\title{
Exotic branes and non-perturbative seven branes
}

\author{
Eduardo Eyras ${ }^{\mathrm{a}}$, Yolanda Lozano ${ }^{\mathrm{b}}$ \\ a Institute for Theoretical Physics, University of Groningen, Nijenborgh 4, \\ 9747 AG Groningen, The Netherlands \\ ${ }^{\mathrm{b}}$ Spinoza Institute, University of Utrecht, Leuvenlaan 4, 3508 TD Utrecht, The Netherlands
}

Received 25 August 1999; accepted 15 December 1999

\begin{abstract}
We construct the effective action of certain exotic branes in the Type II theories which are not predicted by their space-time supersymmetry algebras. We analyze in detail the case of the NS-7B brane, S-dual to the D7-brane, and connected by T-duality to other exotic branes in Type IIA: the KK-6A brane and the KK-8A brane (obtained by reduction of the M-theory Kaluza-Klein monopole and M9-brane, respectively). The NS-7B brane carries charge with respect to the S-dual of the RR 8-form, which we identify as a non-local combination of the electric-magnetic duals of the axion and the dilaton. The study of its effective action agrees with previous results in the literature showing that it transforms as an $S L(2, \mathbb{Z})$ triplet together with the D7-brane. We discuss why this brane is not predicted by the Type IIB space-time supersymmetry algebra. In particular we show that the modular transformation relating the D7 and NS-7B brane solutions can be undone by a simple coordinate transformation in the two-dimensional transverse space, equivalent to choosing a different region to parametrize the $S L(2, \mathbb{Z})$ moduli space. We discuss a similar relation between the D6 and KK-6A branes and the D8 and KK-8A branes. (C) 2000 Elsevier Science B.V. All rights reserved.
\end{abstract}

\section{Introduction}

It is by now well known that the BPS spectra of the Type II and M-theories can be obtained by analyzing the central charges that occur in the corresponding supersymmetry algebra [1,2]. This analysis predicts a 0-brane (the 11-dimensional pp-wave), a 2-brane, a 5-brane, a 6-brane (the Kaluza-Klein monopole) and a 9-brane in M-theory. These branes give rise to the brane-scan of the Type IIA theory, namely the 0-brane, the fundamental string, the NS-5A brane, D $p$-branes with $p$ even, $p=0, \ldots, 8$, the Kaluza-Klein monopole (or KK-5A brane) and the NS-9A brane. All these branes are

E-mail addresses: e.a.eyras@phys.rug.nl (E. Eyras), y.lozano@phys.uu.nl (Y. Lozano). 
predicted as well by the Type IIA supersymmetry algebra and other than the NS-9A brane have been extensively studied in the literature. The NS-9A brane is a space-timefilling brane which may play a role in the construction of the heterotic string as a non-perturbative orientifold of the Type IIA theory (see Ref. [3] for some results in this direction).

From the Type IIA brane-scan, via T-duality, one obtains the brane-scan of the Type IIB theory, also predicted by its space-time supersymmetry algebra. It consists on the 0-brane, the fundamental string, the NS-5B brane, D $p$-branes with $p$ odd, $p=$ $-1,1, \ldots, 9$, the Kaluza-Klein monopole (or KK-5B brane) and the NS-9B brane. These branes occur as multiplets of the $\operatorname{SL}(2, \mathbb{Z})$ symmetry of the theory. Namely the (F1,D1), (NS5B,D5) and (NS9B,D9) branes occur as doublets and the D3-brane, D7-brane and KK-monopole as singlets. The D9-brane and NS-9B brane are space-time-filling branes which are charged with respect to the RR 10-form and its S-dual, an NS-NS 10-form [4], respectively. It is well known that D9-branes play a role in the construction of the Type I theory as an orientifold of Type IIB. Similarly, it was argued in $[3,5]$ that NS-9B branes play a role in the construction of the heterotic $\mathrm{SO}(32)$ theory as a non-perturbative orientifold of Type IIB. In this case 32 NS-9B branes cancel the charge of the orientifold fixed plane associated to the S-dual operation of world-sheet parity reversal.

It has been pointed out however that there exist some brane solutions in the Type IIA and Type IIB theories which are not predicted by their space-time supersymmetry algebras. These branes have been encountered in different contexts. On the one hand, they are required in order to fill up multiplets of BPS states in representations of the U-duality group of M-theory on a $d$-torus [6-10]. On the other, they are predicted by duality from branes that occur as central charges in the space-time supersymmetry algebras.

In particular it is well known that the Kaluza-Klein monopole of M-theory gives rise to the D6-brane after dimensional reduction along its Taub-NUT direction and to the Type IIA KK-monopole after double-dimensional reduction. The third possibility, reducing along a transverse direction different from the Taub-NUT direction, gives rise to a 6-brane in the Type IIA theory which contains as well a Taub-NUT fiber direction $[6,7]$ (we will denote it as a KK-6A brane) but which is not predicted by the Type IIA space-time supersymmetry algebra. The corresponding supergravity solution is described by a harmonic function on $\mathbb{R}^{2} \times S^{1}$ and therefore is logarithmically divergent [7]. This fact was indicated as an explanation for its non-occurrence in the Type IIA space-time supersymmetry algebra [8], given that these algebras are realized as asymptotic symmetries, with flat asymptotic metric. However, it is still striking that the D7-brane, being as well logarithmically divergent, or the D8-brane, linearly divergent, have associated central charges in the Type IIB and Type IIA space-time supersymmetry algebras.

A second brane in the Type IIA theory not predicted either by its space-time supersymmetry algebra occurs when double dimensionally reducing the M9-brane. A single M9-brane has associated a Killing direction which is interpreted as a world-volume direction, and which appears gauged in the effective action $[8,11,12]$. Reduction along this direction gives the D8-brane. On the other hand, reducing along the transverse direction we obtain the NS-9A brane mentioned previously, which contains a gauged direction also interpreted as a world-volume direction. The third possibility consists in reducing along a world-volume direction, different from the Killing direction. This 


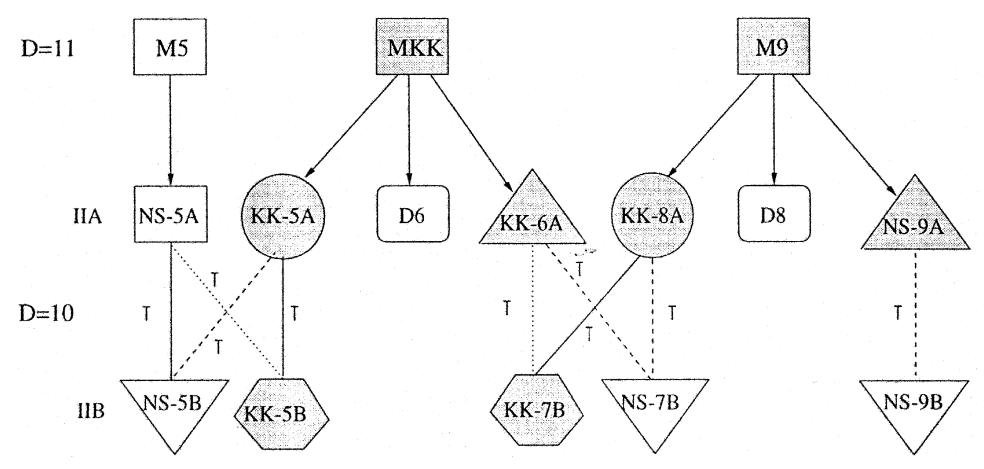

Fig. 1. Exotic branes and T-duality. In this figure we show the relation under T-duality of the different branes considered in this article. We also include the 5-branes and Kaluza-Klein monopoles for comparison of these relations, as well as the M-theory origin of the type IIA branes. With respect to the type IIA branes, we indicate a T-duality along a world-volume direction by a solid line, a T-duality along a Killing direction with a dashed line, and a T-duality along a transverse direction with a dotted line.

predicts a new 8-brane in the Type IIA theory with a gauged direction in its worldvolume, which we denote as a KK-8A brane.

The KK-6A and KK-8A branes are related by T-duality to a new 7-brane in Type IIB (see Fig. 1) which is also predicted by the S-duality symmetry of the theory $[12,13]$. Performing an S-duality transformation in the world-volume effective action of a D p-brane in Type IIB:

$$
S=\int d^{p+1} \xi e^{-\phi} \sqrt{\left|\operatorname{det}\left(g+\left(2 \pi \alpha^{\prime}\right) \mathscr{F}\right)\right|},
$$

with $\mathscr{F}=2 \partial b+\frac{1}{2 \pi \alpha^{\prime}} B^{(2)}$ and $B^{(2)}$ the (pullback of the) NS-NS 2-form, we obtain a $p$-brane with effective action

$$
S=\int d^{p+1} \xi e^{-\left(\frac{p-1}{2}\right) \phi}\left(1+e^{2 \phi}\left(C^{(0)}\right)^{2}\right)^{\frac{p-3}{4}} \sqrt{\left|\operatorname{det}\left(g+\frac{\left(2 \pi \alpha^{\prime}\right) e^{\phi}}{\sqrt{1+e^{2 \phi}\left(C^{(0)}\right)^{2}}} \tilde{\mathscr{F}}\right)\right|}
$$

where $\tilde{\mathscr{F}}=2 \partial c^{(1)}+\frac{1}{2 \pi \alpha^{\prime}} C^{(2)}, C^{(2)}$ is the (pullback of the) RR 2-form and $b \rightarrow-c^{(1)}$ under S-duality. For $p=1$ (1.2) reduces to the Nambu-Goto action of the F-string after dualizing the 1 -form $c^{(1)}$ into a constant scalar ${ }^{1}$. For $p=3$ it does not describe a new 3-brane since world-volume duality of $c^{(1)}$ yields the effective action of the D3-brane, showing that this brane is invariant under S-duality [14-16]. For $p=5,9$ we obtain the kinetic terms of the NS-5B and NS-9B brane effective actions ${ }^{2}$, which form doublets with the D5 and D9 branes respectively. However, for $p=7$ it describes a 7-brane which is not predicted by the Type IIB space-time supersymmetry algebra and which we will denote as an NS-7B brane. This brane cannot be related to the D7-brane by

\footnotetext{
${ }^{1}$ The value of this scalar fixes the number of F-strings after quantization.

${ }^{2}$ See Ref. [17] and Ref. [3], respectively.
} 
world-volume duality. The corresponding supergravity solution, as well as its connections via T-duality with the KK-6A and KK-8A branes in Type IIA, has been studied in [13]. In this reference it is also shown that the NS-7B brane can be cast into a triplet of $S L(2, \mathbb{Z})$ together with the D7-brane. The third charge is the magnetic dilaton charge carried by the 7-brane, and is related to the other two charges upon quantization. Therefore a generic 7-brane can be consistently denoted by two integers. The values of these integers are further restricted by the characterization of the 7-branes by their monodromy matrices or, equivalently, by the type of strings that can end on them [18].

It is the purpose of this paper to study in some more detail these "exotic" branes in the Type IIA and Type IIB theories which are not predicted by their space-time supersymmetry algebras, but are however needed in order to fill up the multiplets of BPS states predicted by the $E_{7}(\mathbb{Z}), E_{8}(\mathbb{Z})$ U-duality groups of M-theory on $T^{7}, T^{8}$ [6-10]. All the exotic branes that we analyze share the property that the tension scales with $1 / g_{s}^{3}$, with $g_{s}$ the string coupling constant, so that they are non-perturbative, i.e. they do not have an obvious interpretation in weakly coupled string theory.

We construct the world-volume effective actions by means of duality transformations on ordinary branes. We mainly focus on the NS-7B brane, and we see from its world-volume effective action that it does not form a doublet with the D7-brane. We identify the 8-form to which this brane is minimally coupled as a non-local combination of the electric-magnetic duals of the axion and the dilaton. This potential needs to be introduced in order to study the behavior of the D7-brane under S-duality. Its field strength however is a local combination of the 9-form field strengths associated to the duals of the axion and dilaton, so that a dual Type IIB supergravity, written as a function of the dual 9-form field strengths, will not depend on it [19,20]. At the level of the solutions we see that the D7 and NS-7B branes are related by a modular transformation, but that this transformation can be undone by a change of coordinates in the transverse space. This means that both branes represent the same physical object in different coordinate systems, and therefore should not have associated independent central charges in the Type IIB space-time supersymmetry algebra. The same kind of transformation identifies the KK-6A brane with the D6 brane, and the KK-8A brane with the D8-brane. This also explains why the KK-6A and KK-8A branes do not appear in the Type IIA space-time supersymmetry algebra.

The paper is organized as follows. In the first four sections we present the effective actions of the exotic branes that we have discussed. We start with the KK-6A brane, which is simply obtained by reducing the M-theory Kaluza-Klein monopole. The NS-7B brane is constructed by T-duality from the KK-6A brane. We see that it is related by S-duality to the D7-brane, where in this duality transformation the RR 8-form is mapped onto another 8-form which is defined as the T-dual of the gravitational field with respect to which the KK-6A brane is charged. We discuss the role played by this field within the Type IIB theory and discuss some properties of the solutions, in particular we show that the D7 and NS-7B brane solutions correspond to local versions of the weak and strong coupling limits of the non-perturbative solution of [21]. In Section 4 we construct the effective action of the KK-8A brane and discuss its interpretation within a non-covariant massive Type IIA supergravity. In Section 5 we give an example of an exotic brane with two gauged isometries which also has a tension $T \sim 1 / g_{s}^{3}$ and is related to the other exotic branes by $\mathrm{T}$-duality. This brane is predicted by U-duality of M-theory on $T^{8}$ and should play a role in the description of eight-di- 
mensional massive supergravity. For all these branes we give an analysis of the brane solitons originated by the boundaries of other branes. Finally in Section 6 we show that a Kaluza-Klein monopole in M-theory with an extra isometric direction can be written as a torus bundle over a two-dimensional base space, and that a modular transformation in the torus can be undone by a simple coordinate transformation in the base space. This allows us to establish an equivalence between the branes that can be derived from the M-theory Kaluza-Klein monopole after reduction and T-duality, in particular between the D6 and the KK-6A branes and the D7 and the NS-7B branes. We also discuss the relation between the KK-8A brane and the D8-brane, using the same construction. Finally, we comment on the origin of this equivalence from the point of view of the M9-brane.

\section{The IIA KK6-brane}

This brane is obtained by reducing the M-theory KK-monopole effective action along a transverse coordinate. In the notation of Ref. [8] it corresponds to the $\left(6,1^{2} ; 3\right)$ brane $^{3}$ predicted by U-duality of M-theory on $T^{7}$. In this section we construct its world-volume effective action and leave for Sections 6 and 7 the discussion about its role within the Type IIA theory.

The action of the M-KK-monopole was constructed in [22,23] and it is given by

$$
\begin{aligned}
\hat{S}_{\mathrm{MKK}}= & -\hat{T}_{\mathrm{MKK}} \int d^{7} \xi|\hat{k}|^{2} \sqrt{\left|\operatorname{det}\left(D_{i} \hat{X}^{\hat{\mu}} D_{j} \hat{X}^{\hat{\nu}} \hat{g}_{\hat{\mu} \hat{\nu}}+l_{p}^{2}|\hat{k}|^{-1} \hat{\mathscr{F}}_{i j}\right)\right|} \\
& +\frac{1}{7 !} l_{p}^{2} \hat{T}_{\mathrm{MKK}} \int d^{7} \xi \epsilon^{i_{1} \ldots i_{7}} \hat{\mathscr{K}}_{i_{1} \ldots i_{7}}^{(7)},
\end{aligned}
$$

with $^{4}$

$$
\hat{\mathscr{F}}=2 \partial \hat{\omega}^{(1)}+l_{p}^{-2} \partial \hat{X}^{\hat{\mu}} \partial \hat{X}^{\hat{\nu}}\left(i_{\hat{k}} \hat{C}\right)_{\hat{\mu} \hat{\nu}},
$$

and

$$
\begin{aligned}
\hat{\mathscr{K}}^{(7)}= & 7 \partial \hat{\omega}^{(6)}-\frac{1}{7} l_{p}^{-2}\left(i_{\hat{k}} \hat{N}\right)+3\left(i_{\hat{k}} \hat{\tilde{C}}\right) \hat{\mathscr{F}}-5 l_{p}^{-2} D \hat{X}^{\hat{\mu}} D \hat{X}^{\hat{\nu}} D \hat{X}^{\hat{\rho}} \hat{C}_{\hat{\mu} \hat{\nu} \hat{\rho}}\left(i_{\hat{k}} \hat{C}\right)\left(i_{\hat{k}} \hat{C}\right) \\
& -30 D \hat{X}^{\hat{\mu}} D \hat{X}^{\hat{\nu}} D \hat{X}^{\hat{\rho}} \hat{C}_{\hat{\mu} \hat{\nu} \hat{\rho}}\left(i_{\hat{k}} \hat{C}\right) \partial \hat{\omega}^{(1)}-60 l_{p}^{2} D \hat{X}^{\hat{\mu}} D \hat{X}^{\hat{\nu}} D \hat{X}^{\hat{\rho}} \hat{C}_{\hat{\mu} \hat{\nu} \hat{\rho}} \partial \hat{\omega}^{(1)} \partial \hat{\omega}^{(1)} \\
& \left.-120 l_{p}^{4} \hat{A} \partial \hat{\omega}^{(1)} \partial \hat{\omega}^{(1)} \partial \hat{\omega}^{(1)}\right\} .
\end{aligned}
$$

This action reflects the fact that the Taub-NUT space of the monopole is isometric in its Taub-NUT direction, so that the transverse space is effectively three dimensional. The

\footnotetext{
${ }^{3}$ In the notation of [8] the first entry gives the number of ordinary spatial directions, the entries of the form $m^{n}$ indicate that there are $m$ spatial directions which are gauged in the effective action and whose radii have $n$th power. Finally the last entry gives the power of the inverse of the string coupling constant.

${ }^{4} i_{\hat{k}} \hat{P}$ denotes the interior product of a field $\hat{P}$ with the Killing vector.
} 
extra degree of freedom is eliminated by imposing a Killing isometry in the Taub-NUT direction,

$$
\delta \hat{X}^{\hat{\mu}}=-\hat{\sigma}^{(0)} \hat{k}^{\hat{\mu}},
$$

which is gauged through the introduction of the dependent gauge field $\hat{A^{5}}{ }^{5}$

$$
\hat{A}=|\hat{k}|^{-2} \partial \hat{X}^{\hat{\mu}} \hat{k}_{\hat{\mu}}
$$

such that $\hat{k}_{\hat{\mu}} D \hat{X}^{\hat{\mu}}=0$, with $D \hat{X}^{\hat{\mu}}$ the covariant derivative,

$$
D \hat{X}^{\hat{\mu}}=\partial \hat{X}^{\hat{\mu}}+\hat{A} \hat{k}^{\hat{\mu}} .
$$

See Ref. [22,23] for more details. $\hat{\tilde{C}}$ is the 3-form field of 11-dimensional supergravity, $\hat{\tilde{C}}$ its electric-magnetic dual and $\hat{N}$ is the dual of the Killing vector $\hat{k}_{\hat{\mu}}$ considered as a 1 -form. It is therefore a gravitational field. The presence of covariant derivatives and contractions with the Killing vector assures invariance under local isometric transformations: $\delta \hat{X}^{\hat{\mu}}=-\hat{\sigma}^{(0)}(\xi) \hat{k}^{\hat{\mu}}$. The field content is then that of the seven-dimensional vector multiplet, involving three scalars and one vector. The gauge transformations of the target space and world-volume fields involved in the action can be found in [23].

In order to obtain the KK-6A brane effective action we perform a direct dimensional reduction along a coordinate $y$ different from the Taub-NUT direction. Accordingly, the transverse space of the KK-6A brane still contains an isometric Taub-NUT direction, which remains gauged in the action after the reduction. The field content is again that of the seven-dimensional vector multiplet, taking into account the additional scalar coming from the reduction.

The background fields reduce as follows:

$$
\begin{aligned}
& \left(i_{\hat{k}} \hat{N}\right)_{\mu_{1} \ldots \mu_{7}}=\left(i_{k} N^{(8)}\right)_{\mu_{1} \ldots \mu_{7}}, \\
& \left(i_{k} \hat{N}\right)_{\mu_{1} \ldots \mu_{6} y}=-\left(i_{k} N^{(7)}\right)_{\mu_{1} \ldots \mu_{6}} \text {, } \\
& \left(i_{\hat{k}} \hat{\tilde{C}}\right)_{\mu_{1} \ldots \mu_{5}}=-\left(i_{k} B^{(6)}\right)_{\mu_{1} \ldots \mu_{5}}, \\
& \left(i_{\hat{k}} \hat{\tilde{C}}\right)_{\mu_{1} \ldots \mu_{4} y}=-\left(i_{k} C^{(5)}\right)_{\mu_{1} \ldots \mu_{4}}+2 C_{\left[\mu_{1} \mu_{2} \mu_{3}\right.}^{(3)}\left(i_{k} B^{(2)}\right)_{\left.\mu_{4}\right]} \\
& +3\left(i_{k} C^{(3)}\right)_{\left[\mu_{1} \mu_{2}\right.} B_{\left.\mu_{3} \mu_{4}\right]}^{(2)}, \\
& \left(i_{\hat{k}} \hat{C}\right)_{\mu_{1} \mu_{2}}=\left(i_{k} C^{(3)}\right)_{\mu_{1} \mu_{2}} \text {, } \\
& \left(i_{\hat{k}} \hat{C}\right)_{\mu y}=-\left(i_{k} B^{(2)}\right)_{\mu} \text {. }
\end{aligned}
$$

$N^{(7)}$ is the electric-magnetic dual of the Killing vector $k_{\mu}, i_{k} N^{(7)}$ being the field to which the IIA KK-monopole couples minimally [23]. Its gauge transformation rule can be found in that reference. The field $N^{(8)}$ comes from the reduction of $\hat{N}$, electric-magnetic dual of $\hat{k}_{\hat{\mu}}$, and must be dual to the modulus of the Killing vector (see Section 7 for a discussion on this point). Its gauge transformation rule can be found in Appendix

\footnotetext{
${ }^{5}$ In our notation $|\hat{k}|^{2}=-\hat{k}^{\hat{\mu}} \hat{k}^{\hat{\nu}} \hat{g}_{\hat{\mu} \hat{\nu}}$.
} 
A. Both fields are therefore purely gravitational. $C^{(p)}$ denotes a $p$-form RR field, $B^{(2)}$ the NS-NS 2-form and $B^{(6)}$ its electric-magnetic dual. The notation for their gauge transformation rules is that of Ref. [17].

We also have

$$
\begin{aligned}
& \hat{\mathscr{F}}=\mathscr{H}^{(2)}, \\
& \hat{A}=A-\left(2 \pi \alpha^{\prime}\right) \frac{e^{2 \phi}\left(i_{k} C^{(1)}\right)}{|k|^{2}+e^{2 \phi}\left(i_{k} C^{(1)}\right)^{2}} \mathscr{G}^{(1)},
\end{aligned}
$$

where

$$
\begin{aligned}
& \mathscr{H}^{(2)}=2 \partial d^{(1)}+\frac{1}{2 \pi \alpha^{\prime}}\left(i_{k} C^{(3)}\right)-2\left(i_{k} B^{(2)}\right) \partial c^{(0)}, \\
& \mathscr{G}^{(1)}=\partial c^{(0)}+\frac{1}{2 \pi \alpha^{\prime}}\left(D X C^{(1)}\right),
\end{aligned}
$$

and

$$
c^{(0)}=y /\left(2 \pi \alpha^{\prime}\right), \quad d^{(1)}=\hat{\omega}^{(1)} .
$$

The result for the dimensionally reduced action is

$S_{\mathrm{KK} 6 \mathrm{~A}}$

$$
\begin{aligned}
= & -T_{\mathrm{KK} 6 \mathrm{~A}} \int d^{7} \xi e^{-3 \phi}|k|^{2}\left(1+e^{2 \phi}|k|^{-2}\left(i_{k} C^{(1)}\right)^{2}\right) \\
& \times\left\{\operatorname { d e t } \left(D_{i} X^{\mu} D_{j} X^{\nu} g_{\mu \nu}-\frac{\left(2 \pi \alpha^{\prime}\right)^{2} e^{2 \phi}}{1+e^{2 \phi}|k|^{-2}\left(i_{k} C^{(1)}\right)^{2}} \mathscr{G}_{i}^{(1)} \mathscr{G}_{j}^{(1)}\right.\right. \\
& \left.\left.+\frac{\left(2 \pi \alpha^{\prime}\right)|k|^{-1} e^{\phi}}{\sqrt{1+e^{2 \phi}|k|^{-2}\left(i_{k} C^{(1)}\right)^{2}}} \mathscr{H}_{i j}^{(2)}\right) \mid\right\}^{1 / 2} \\
& +\frac{1}{7 !}\left(2 \pi \alpha^{\prime}\right) T_{\mathrm{KK} 6 \mathrm{~A}} \int d^{7} \xi \epsilon^{i_{1} \ldots i_{7}} \mathscr{K}_{i_{1} \ldots i_{7}}^{(7)},
\end{aligned}
$$

where $T_{\mathrm{KK} 6 \mathrm{~A}}=\hat{T}_{\mathrm{MKK}}$ and $\mathscr{K}^{(7)}$ is given by

$$
\begin{aligned}
\mathscr{K}^{(7)}= & 7\left\{\partial \omega^{(6)}-\frac{1}{7\left(2 \pi \alpha^{\prime}\right)}\left(i_{k} N^{(8)}\right)+\left(i_{k} N^{(7)}\right) \partial c^{(0)}-3\left(i_{k} B^{(6)}\right) \mathscr{H}^{(2)}\right. \\
& -15\left(2 \pi \alpha^{\prime}\right)\left(i_{k} C^{(5)}\right) \partial c^{(0)} \mathscr{H}^{(2)}+30\left(2 \pi \alpha^{\prime}\right) C^{(3)}\left(i_{k} B^{(2)}\right) \partial c^{(0)} \mathscr{H}^{(2)} \\
& +45\left(2 \pi \alpha^{\prime}\right)\left(i_{k} C^{(3)}\right) B^{(2)} \partial c^{(0)} \mathscr{H}^{(2)}-\frac{5}{2 \pi \alpha^{\prime}} C^{(3)}\left(i_{k} C^{(3)}\right)^{2}
\end{aligned}
$$




$$
\begin{aligned}
& +20 C^{(3)}\left(i_{k} C^{(3)}\right)\left(i_{k} B^{(2)}\right) \partial c^{(0)}-30\left(2 \pi \alpha^{\prime}\right) C^{(3)} \mathscr{H}^{(2)} \partial d^{(1)} \\
& -15 B^{(2)} \partial c^{(0)}\left(i_{k} C^{(3)}\right)^{2}-90\left(2 \pi \alpha^{\prime}\right)^{2} B^{(2)} \partial c^{(0)} \mathscr{H}^{(2)} \partial d^{(1)} \\
& \left.-15\left(2 \pi \alpha^{\prime}\right)^{2}\left(A-\frac{\left(2 \pi \alpha^{\prime}\right) e^{2 \phi}\left(i_{k} C^{(1)}\right)}{|k|^{2}+e^{2 \phi}\left(i_{k} C^{(1)}\right)^{2}}\right)\left(\mathscr{G}^{(1)}\right)\left(\mathscr{H}^{(2)}\right)^{3}\right\} .
\end{aligned}
$$

The covariant derivative is defined as

$$
D X^{\mu}=\partial X^{\mu}+A k^{\mu}
$$

with $A$ given by

$$
A=|k|^{-2} \partial X^{\mu} k_{\mu} \text {. }
$$

Finally, the effective tension of this brane is given by

$$
\mathscr{T}_{\mathrm{KK} 6 \mathrm{~A}}=\frac{R_{k}^{2}}{(2 \pi)^{6} g_{s}^{3} l_{s}^{9}},
$$

where $R_{k}$ is the radius of the compact direction associated to the Taub-NUT isometry and $l_{s}$ is the string length.

We find that the world-volume field content is that of the seven-dimensional vector multiplet, namely a scalar $c^{(0)}$, a vector $d^{(1)}$ and a 6 -form $\omega^{(6)}$ playing the role of tension of the brane. These fields can be given an interpretation in terms of solitons in the brane. In general, world-volume fields coupled to a given brane effective action describe soliton solutions in its world-volume. For branes with a Killing direction, the world-volume fields enter in the field strengths as

$$
\mathscr{K}^{(p)}=p \partial \omega^{(p-1)}+\frac{1}{2 \pi \alpha^{\prime}}\left(i_{k} C^{(p+1)}\right)+\ldots,
$$

from which we can conclude that $\omega^{(p-1)}$ couples to a $(p-2)$-brane soliton describing the boundary of a $p$-brane ending on the monopole, with one of its world-volume directions wrapped around the Killing direction [17]. Therefore $c^{(0)}$ must couple to a 4-brane soliton (through its world-volume dual), $d^{(1)}$ to a 0-brane and a 3-brane solitons, and $\omega^{(6)}$ to a domain-wall soliton. The corresponding configurations of branes ending on the KK-6A brane can be associated to some of the intersections of the KK-6A brane with other Type IIA branes. These were classified in [24]. We find

- $\quad$ The intersections $(0 \mid \mathrm{D} 2, \mathrm{KK} 6 \mathrm{~A})$ and $(0 \mid \mathrm{F} 1, \mathrm{KK} 6 \mathrm{~A})$ are associated to a 0-brane soliton coupled to $d^{(1)}$. These configurations are obtained from the intersection $(0 \mid \mathrm{M} 2, \mathrm{MKK})$ in M-theory.

- $\quad(3 \mid \mathrm{D} 4, \mathrm{KK} 6 \mathrm{~A})$ and (3|NS5A,KK6A) are associated to a 3-brane soliton coupled magnetically to $d^{(1)}$. They are obtained from the configuration (3|M5,MKK).

(5|NS5A,KK6A) represents an NS-5A brane embedded in the KK-6A and it is associated to a domain-wall soliton which couples to $\omega^{(6)}$. It is obtained from the configuration (5|M5,MKK).

- $\quad$ Further, we find three configurations that can give rise to a 4-brane soliton. The cases $(4 \mid \mathrm{KK} 6 \mathrm{~A}, \mathrm{KK} 6 \mathrm{~A})^{a}$ and $(4 \mid \mathrm{KK} 5 \mathrm{~A}, \mathrm{KK} 6 \mathrm{~A})^{b}$ couple magnetically to the embedding scalars and (4|D6,KK6A) couples magnetically to the form $c^{(0)}$. The 
index $a(b)$ indicates that the isometries of both objects lie in the same (a different) direction. These configurations are obtained from the configurations $(4 \mid \mathrm{MKK}, \mathrm{MKK})^{a, b}$ in M-theory through reduction along a transverse direction [24].

\section{The IIB NS-7 brane}

As we have mentioned in Section 1, the NS-7B brane can be obtained by T-duality from the KK-6A brane. The T-duality transformation takes place along the Taub-NUT direction of the KK-6A brane and the result is the double-dimensional reduction of the NS-7B brane. This brane plays a role in the construction of the massive nine-dimensional Type II supergravity of [13], which is manifestly invariant under $S L(2, \mathbb{R})$. This theory contains three mass parameters, only two of which are independent upon quantization, and they have their origin in the presence of D7 and NS-7B branes in the background. Also, as we discuss at the end of this section, the orientifold constructions of the Type IIB theory on $T^{2}$ describing certain branches of F-theory on $\mathrm{K} 3$ require the introduction of NS-7B space-time filling branes to cancel the charge of the orientifold fixed planes at strong coupling.

\subsection{The NS-7B brane effective action}

In this section we construct the world-volume effective action of the NS-7B brane by performing a T-duality on the KK-6A brane effective action. In the next subsections we show that it is connected to the D7-brane by an S-duality transformation and discuss in some extent the role that this brane plays within the Type IIB theory.

The result of a T-duality transformation in the effective action of the KK-6A brane along its Taub-NUT direction is the (double-dimensional reduction of the) action,

$$
\begin{aligned}
S_{\mathrm{NS} 7}= & -T_{\mathrm{NS} 7} \int d^{8} \xi e^{-3 \phi}\left(1+e^{2 \phi}\left(C^{(0)}\right)^{2}\right) \sqrt{\left|\operatorname{det}\left(g+\frac{\left(2 \pi \alpha^{\prime}\right) e^{\phi}}{\sqrt{1+e^{2 \phi}\left(C^{(0)}\right)^{2}}} \tilde{\mathscr{F}}\right)\right|} \\
& +\frac{1}{8 !}\left(2 \pi \alpha^{\prime}\right) T_{\mathrm{NS} 7} \int d^{8} \xi \epsilon^{i_{1} \ldots i_{8}} \tilde{\mathscr{G}}_{i_{1} \ldots i_{8}}^{(8)},
\end{aligned}
$$

where $T_{\mathrm{NS} 7}$ is such that $T_{\mathrm{NS} 7} \int d \xi^{8}=T_{\mathrm{KK} 6 \mathrm{~A}}$ and $\tilde{\mathscr{G}}^{(8)}$ is given by

$$
\begin{aligned}
\tilde{\mathscr{G}}^{(8)}= & \left\{8 \partial \tilde{C}^{(7)}-\frac{1}{2 \pi \alpha^{\prime}} \tilde{C}^{(8)}+\frac{1}{2} \frac{8 !}{6 !} B^{(6)} \tilde{\mathscr{F}}+\frac{7 !}{4 !}\left(2 \pi \alpha^{\prime}\right) C^{(4)} \tilde{\mathscr{F}}^{2}\right. \\
& +\frac{1}{2} \frac{7 !}{3 !}\left(2 \pi \alpha^{\prime}\right)^{2} B^{(2)} \tilde{\mathscr{F}}^{3}-\frac{1}{2} \frac{7 !}{4 !}\left(2 \pi \alpha^{\prime}\right)^{3} \frac{C^{(0)}}{\left(C^{(0)}\right)^{2}+e^{-2 \phi}} \tilde{\mathscr{F}}^{4} \\
& -\frac{7 !}{8}\left(2 \pi \alpha^{\prime}\right) B^{(2)} C^{(2)} \tilde{\mathscr{F}}^{2}+\frac{7 !}{8} B^{(2)}\left(C^{(2)}\right)^{2} \tilde{\mathscr{F}} \\
& \left.-\frac{1}{2} \frac{7 !}{3 !} \frac{1}{2 \pi \alpha^{\prime}} B^{(2)}\left(C^{(2)}\right)^{3}\right\} .
\end{aligned}
$$


The T-duality transformation rules of the background fields that couple to the KK-6A brane can be found for instance in [17], whereas the transformation rule of the gravitational field $N^{(8)}$ has to be worked out, with the result that it transforms as

$$
\begin{aligned}
\left(i_{k} N^{(8)}\right)_{\mu_{1} \ldots \mu_{7}}^{\prime}= & \tilde{C}_{\mu_{1} \ldots \mu_{7} z}^{(8)}-7\left(B_{\left[\mu_{1} \ldots \mu_{6}\right.}^{(6)}-6 B_{\left[\mu_{1} \ldots \mu_{5} z\right.}^{(6)} \frac{g_{\mu_{6} z}}{g_{z z}}\right) C_{\left.\mu_{7}\right] z}^{(2)} \\
& +35 C_{\left[\mu_{1} \ldots \mu_{3} z\right.}^{(4)} C_{\mu_{4} \mu_{5}}^{(2)}\left(C_{\left.\mu_{6} \mu_{7}\right]}^{(2)}+4 \frac{g_{\mu_{6} z}}{g_{z z}} C_{\left.\mu_{7}\right] z}^{(2)}\right) \\
& -105\left(2 B_{\left[\mu_{1} \mu_{2}\right.}^{(2)}-B_{\left[\mu_{1} z\right.}^{(2)} \frac{g_{\mu_{2} z}}{g_{z z}}\right) C_{\mu_{3} z}^{(2)}\left(C^{(2)}\right)_{\left.\mu_{4} \ldots \mu_{7}\right]}^{2}
\end{aligned}
$$

This defines a new field $\tilde{C}^{(8)}$ in Type IIB, which as we will discuss below is the S-dual of the RR 8-form.

The world-volume fields transform as follows:

$$
c^{(0)^{\prime}}=-c_{\sigma}^{(1)}, \quad d^{(1)^{\prime}}=-c^{(1)}
$$

so that

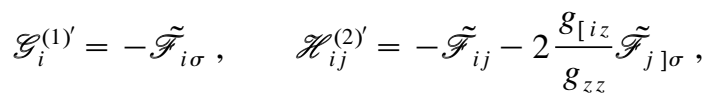

with

$$
\tilde{\mathscr{F}}=2 \partial c^{(1)}+\frac{1}{2 \pi \alpha^{\prime}} C^{(2)}
$$

the field strength associated to the RR 2-form. The tensions transform simply as

$$
\omega_{i_{1} \ldots i_{6}}^{(6)^{\prime}}=\tilde{c}_{i_{1} \ldots i_{6} \sigma}^{(7)} .
$$

From (3.2) we see that the NS-7B brane is minimally coupled to the field $\tilde{C}^{(8)}$, arising from the T-duality transformation (3.3). The interpretation of this new field in the IIB theory is discussed in Subsection 3.2 Eqs. (3.1) and (3.2) also identify the strings that can end on the brane as D-strings, so that this brane corresponds to the $(0,1) 7$-brane in the notation of [18].

Before comparing with the D7-brane effective action let us analyze the possible soliton solutions that can occur in the world-volume of this brane. Its world-volume field content consists on $c^{(1)}$ and $\tilde{c}^{(7)}$, playing the role of tension of the brane, as well as the embedding scalars. They couple to the soliton configurations:

- $\quad(0 \mid \mathrm{D} 1, \mathrm{NS} 7 \mathrm{~B})$ gives a 0 -brane soliton coupled to $c^{(1)}$. It is related to the configuration (0|D2,KK6A) by T-duality.

- $\quad(4 \mid \mathrm{D} 5, \mathrm{NS} 7 \mathrm{~B})$ gives a 4-brane soliton coupled magnetically to $c^{(1)}$. It is related by T-duality to (4|D6,KK6A).

- $\quad$ (5|NS7B,NS7B) and (5|KK5B,NS7B) give a 5-brane soliton magnetically coupled to the embedding scalars. They are related by $\mathrm{T}$-duality to $(4 \mid \mathrm{KK} 6 \mathrm{~A}, \mathrm{KK} 6 \mathrm{~A})^{a}$, and $(4 \mid \mathrm{KK} 5 \mathrm{~A}, \mathrm{KK} 6 \mathrm{~A})^{b},(5 \mid \mathrm{NS} 5 \mathrm{~A}, \mathrm{KK} 6 \mathrm{~A})$ respectively. 
- $\quad$ Note that the presence of $\tilde{c}^{(7)}$ as a dynamical tension is not entirely justified. The existence of such a dynamical tension implies that the NS-7B brane can be open [25], hence the NS-7B (and by S-duality, the D7-brane) would have boundaries. However, this would not define consistently the monodromies associated to these 7-branes.

\subsection{D7-branes, NS7-branes and S-duality}

The effective action that we have found for the NS-7B brane is related by S-duality to the effective action of the D7-brane:

$$
S_{\mathrm{D} 7}=-T_{\mathrm{D} 7} \int d^{8} \xi\left\{e^{-\phi} \sqrt{\left|\operatorname{det}\left(g+\left(2 \pi \alpha^{\prime}\right) \mathscr{F}\right)\right|}+\frac{\left(2 \pi \alpha^{\prime}\right)}{8 !} \epsilon^{i_{1} \ldots i_{8} \mathscr{G}_{i_{1} \ldots i_{8}}^{(8)}}\right\},
$$

with $\mathscr{F}=2 \partial b+\frac{1}{2 \pi \alpha^{\prime}} B^{(2)}$ and $\mathscr{G}^{(8)}$ given by

$$
\begin{aligned}
\mathscr{G}^{(8)}= & \left\{8 \partial c^{(7)}+\frac{1}{2 \pi \alpha^{\prime}} C^{(8)}-\frac{1}{2} \frac{8 !}{6 !} C^{(6)} \mathscr{F}+\frac{7 !}{4 !}\left(2 \pi \alpha^{\prime}\right) C^{(4) \mathscr{F}^{2}}\right. \\
& -\frac{1}{2} \frac{7 !}{3 !}\left(2 \pi \alpha^{\prime}\right)^{2} C^{(2)} \mathscr{F}^{3}+\frac{1}{2} \frac{7 !}{4 !}\left(2 \pi \alpha^{\prime}\right)^{3} C^{(0)} \mathscr{F}^{4}+\frac{7 !}{8}\left(2 \pi \alpha^{\prime}\right) C^{(2)} B^{(2)} \mathscr{F}^{2} \\
& \left.-\frac{7 !}{8} C^{(2)}\left(B^{(2)}\right)^{2} \mathscr{F}+\frac{1}{2} \frac{7 !}{3 !} \frac{1}{2 \pi \alpha^{\prime}}\left(B^{(2)}\right)^{3} C^{(2)}\right\}
\end{aligned}
$$

yields (3.1) after an S-duality transformation,

$$
\begin{aligned}
& C^{(0)} \rightarrow \frac{-C^{(0)}}{\left(C^{(0)}\right)^{2}+e^{-2 \varphi}}, \quad e^{-\varphi} \rightarrow \frac{e^{-\varphi}}{\left(C^{(0)}\right)^{2}+e^{-2 \varphi}} \\
& C^{(2)} \rightarrow B^{(2)}, \quad B^{(2)} \rightarrow-C^{(2)}, \\
& C^{(6)} \rightarrow B^{(6)}, \quad B^{(6)} \rightarrow-C^{(6)}, \\
& C^{(4)} \rightarrow C^{(4)}, \\
& C^{(8)} \rightarrow-\tilde{C}^{(8)}, \\
& \tilde{C}^{(8)} \rightarrow-C^{(8)},
\end{aligned}
$$

together with

$$
\begin{array}{llllll}
b & \rightarrow & -c^{(1)}, & c^{(7)} & \rightarrow & \tilde{c}^{(7)}, \\
c^{(1)} & \rightarrow & b, & \tilde{c}^{(7)} & \rightarrow & c^{(7)},
\end{array}
$$

for the world-volume fields.

One can check as well that the soliton configurations that we have found for the NS-7B brane are mapped under S-duality to the usual intersections involving the D7-brane.

The transformation rules for $C^{(8)}$ and $\tilde{C}^{(8)}$ in (3.10) show that the D7 and NS-7B branes do not form a doublet under S-duality. They imply in particular that after two S-duality transformations each WZ term is mapped onto itself, whereas for doublets $S^{2}=-1$. This observation from the world-volume effective actions is in agreement with 
the results of [13], where it is shown that Type IIB 7-branes transform as triplets under $S L(2, \mathbb{Z})$. We now elaborate on this point.

Consider the conserved currents (in Einstein frame) associated to the RR scalar and dilaton of the Type IIB theory:

$$
j^{(0)}=e^{2 \phi} d C^{(0)}, \quad j^{(\phi)}=e^{2 \phi} d|\lambda|^{2} .
$$

Here $\lambda=C^{(0)}+i e^{-\phi}$ is the axion-dilaton modulus. These currents define the electricmagnetic duals of the RR scalar and dilaton, namely the fields $C^{(8)}$, to which the D7-brane couples minimally, and a certain 8 -form $B^{(8)}$, which in principle could be associated to another 7-brane in the theory. There is a third conserved current [13] which can be obtained as a combination of the previous two with non-constant coefficients:

$$
j=-C^{(0)} j^{(\phi)}+|\lambda|^{2} j^{(0)},
$$

to which we can associate as well a dual field. The transformation rules of these currents under the $S$ and $T$ generators of the $\operatorname{SL}(2, \mathbb{Z})$ group are [13]

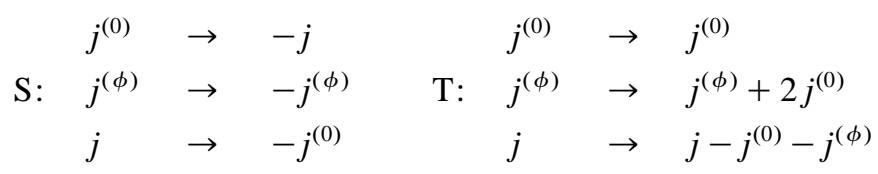

The transformations of $j^{(0)}$ and $j$ under $\mathrm{S}$ suggest that the 8-form associated to $j$ should be the S-transformed of $C^{(8)}$, namely $\tilde{C}^{(8)}$. Indeed, with this identification the S-duality transformations of the 8 -forms become

$$
\mathrm{S}: \quad \begin{array}{lll}
C^{(8)} & \rightarrow & -\tilde{C}^{(8)} \\
\tilde{C}^{(8)} & \rightarrow & -C^{(8)} \\
B^{(8)} & \rightarrow & -B^{(8)}
\end{array}
$$

which agree with the transformation rules for $C^{(8)}$ and $\tilde{C}^{(8)}$ in (3.10). We see that a 7-brane magnetically coupled to the dilaton would transform as a singlet under S-duality, and that the 8-forms $C^{(8)}$ and $\tilde{C}^{(8)}$ do not transform as a doublet. In fact, the 8-forms transform as a triplet under S-duality, and in general, under the $S L(2, \mathbb{Z})$ symmetry group:

$$
\mathscr{M}=\left(\begin{array}{cc}
B^{(8)} & 2 \tilde{C}^{(8)} \\
2 C^{(8)} & -B^{(8)}
\end{array}\right) \rightarrow \Lambda \mathscr{M} \Lambda^{-1}, \quad \forall \Lambda \in S L(2, \mathbb{Z}) .
$$

The field $\tilde{C}^{(8)}$ becomes necessary when we try to map $C^{(8)}$ onto a local field under S-duality, but of course the three 8 -forms are not independent, since they are related through (3.13), which in terms of 8-forms reads

$$
d \tilde{C}^{(8)}=-C^{(0)} d B^{(8)}+|\lambda|^{2} d C^{(8)} .
$$

Therefore $\tilde{C}^{(8)}$ is not interpreted as an independent field in the theory. A dual Type IIB supergravity, written as a function of the dual potentials, will depend only on two of the 
three 9-form field strengths ${ }^{6}$, which is consistent with the results of [20] and [19] ${ }^{7}$. This relation only becomes local for constant axion-dilaton modulus:

$$
\tilde{C}^{(8)}=-C^{(0)} B^{(8)}+|\lambda|^{2} C^{(8)} .
$$

In order to clarify further the role of this three 8-form potentials let us consider the solution representing a single D7-brane located at the origin of the transverse space $[13,26]$ (in string frame):

$$
d s^{2}=H^{-1 / 2} d s_{1,7}^{2}-H^{1 / 2} d \omega d \bar{\omega}, \quad \lambda=\frac{1}{2 \pi i} \log \omega,
$$

where the harmonic function is given by $H=-\frac{1}{2 \pi} \log |\omega|$ with $\omega=x_{2}+i x_{1}$, and $x_{1}, x_{2}$ the two transverse coordinates. The RR charge of this brane can be computed as

$$
p=\oint_{\gamma}^{*} d C^{(8)}
$$

where $\gamma$ is a circle around the origin in the transverse space. A 7-brane with a non-vanishing value for the dilaton carries as well magnetic dilaton charge, which is computed as

$$
s=\oint_{\gamma}^{*} d B^{(8)}
$$

Moreover, through (3.17) it also carries $\tilde{C}^{(8)}$-charge,

$$
q=\oint_{\gamma} * d \tilde{C}^{(8)}=\oint_{\gamma}\left(-C^{(0) *} d B^{(8)}+|\lambda|^{2 *} d C^{(8)}\right) .
$$

The values of the three charges can be computed with the only input of the axion-dilaton modulus associated to the solution. The quantization of the charges is obtained by imposing that $e^{Q}$ with

$$
Q=\left(\begin{array}{cc}
s & 2 p \\
2 q & -s
\end{array}\right)
$$

belongs to $S L(2, \mathbb{Z})$. Imposing this condition it is possible to see that the three charges can be obtained from only two independent integers (see subsection 5.1 in Ref. [13]) whose values specify uniquely the 7-brane charges. It also implies that it is not possible to have a solution with vanishing $p$ and $q$ charges and non-vanishing $s$, which is consistent with the fact that a 7-brane carrying only magnetic dilaton charge cannot exist, given that $(3.21)$ vanishes for $C^{(0)}=0$. Taking into account that 7-branes with the

\footnotetext{
${ }^{6}$ As explained in [19] this formulation will depend as well on the original fields through the Chern-Simons forms in the definition of the curvatures.

${ }^{7}$ In this last reference three different 9-form field strengths appear in the formulation of Type IIB supergravity in terms of original and dual fields due to the fact that the action is manifestly SU(1,1) invariant with the U(1) invariance not being fixed.
} 
same monodromy matrix are equivalent, the charge matrix is further restricted to have the form [18]

$$
\Lambda\left(\begin{array}{ll}
1 & 1 \\
0 & 1
\end{array}\right) \Lambda^{-1}, \quad \forall \Lambda \in S L(2, \mathbb{Z}) .
$$

If we compute the values of the charges carried by the D7-brane solution above,

$$
\begin{aligned}
& p=\oint_{\gamma} e^{2 \phi} d C^{(0)} \\
& q=\oint_{\gamma} e^{2 \phi}\left(-C^{(0)} d|\lambda|^{2}+|\lambda|^{2} d C^{(0)}\right) \\
& s=\oint_{\gamma} e^{2 \phi} d|\lambda|^{2}
\end{aligned}
$$

we find that $p=s=4 \pi^{2}(\log r)^{-2}$ and $q=1-p / 3$, where we have parametrized $\omega=r e^{i \theta}$. Therefore asymptotically $p$ and $s$ vanish whereas the $q$ charge tends to a constant value ${ }^{8}$. This is consistent with the fact that asymptotically the string coupling constant vanishes so that we are in a weak coupling regime, in which $\tilde{C}^{(8)}$ is not well defined. In the strong coupling limit the solution that makes sense is the S-dual of the D7-brane solution above, which has the following form [13]:

$$
\begin{aligned}
& d \tilde{s}^{2}=\left(H^{2}+\theta^{2} /(2 \pi)^{2}\right)^{1 / 2}\left[H^{-1 / 2} d s_{1,7}^{2}-H^{1 / 2} d \omega d \bar{\omega}\right], \\
& \tilde{\lambda}=-2 \pi i / \log \omega .
\end{aligned}
$$

Here the harmonic function is given by $H=-\frac{1}{2 \pi} \log |\omega|$, as before. This solution carries as well $(\tilde{p}, \tilde{q}, \tilde{s})$ charges, which in the Einstein frame take the values: $\tilde{q}=\tilde{s}=$ $-4 \pi^{2}(\log r)^{-2}, \tilde{p}=-(1+\tilde{q} / 3)$, i.e. they are related to the charges of the D7-brane solution by the $\mathrm{S}$-duality transformation in (3.14). In this case it is the $\tilde{p}$ charge that has a constant value at infinity, whereas $\tilde{q}=\tilde{s}=0$.

Let us now clarify the relation between the D7 and NS-7B brane solutions that we have just considered and the finite energy 7-brane solution of [21] ${ }^{9}$. In order to construct a finite energy 7-brane solution, where the modulus $\lambda$ depends holomorphically on the transverse space, it is crucial to allow $\lambda$ to have an ambiguity under $\operatorname{SL}(2, \mathbb{Z})$ transformations [21,28]. Then the solution is constructed in such a way that the axion-dilaton modulus parametrizes the fundamental region of $S L(2, \mathbb{Z})$, therefore all choices related by an $S L(2, \mathbb{Z})$ transformation have been identified, and there is a one to one mapping from the fundamental region of $\operatorname{SL}(2, \mathbb{Z})$ and the complex plane. This is achieved by fulfilling the condition $\bar{\partial} \lambda(\omega, \bar{\omega})=0$, derived from the equations of motion, implicitly through the elliptic modular function:

$$
j(\lambda(\omega))=\frac{1}{\omega}
$$

\footnotetext{
${ }^{8}$ In string frame $p=s=1$ and $q=-\frac{1}{3}+1 / g_{s t}^{2}$. However, the Einstein frame is more useful in order to study the S-duality properties of the solution.

${ }^{9}$ We will adopt however the conventions in [27].
} 
with $j$ given by

$$
j(\tau)=\frac{\left(\theta_{2}(\tau)^{8}+\theta_{3}(\tau)^{8}+\theta_{4}(\tau)^{8}\right)^{3}}{\eta(\tau)^{24}},
$$

instead of simply taking $\lambda$ as a function of $\omega . \theta_{i}$ are the Jacobi $\theta$-functions and $\eta$ is the Dedekind $\eta$ function:

$$
\eta(\tau)=q^{1 / 24} \prod_{n=1}^{\infty}\left(1-q^{n}\right), \quad q=e^{2 \pi i \tau} .
$$

The metric associated to this solution has a deficit angle of $\pi / 6$ near $\omega=0$, so that taking precisely 24 7-branes the plane is curled up into a 2 -sphere and one ends up with a compact manifold. This compactification can equivalently be described as an elliptically fibered K3, with $S^{2}$ the base of the fiber and $\lambda$ the $\tau$ parameter of the fiber torus. This defines F-theory compactified on K3, and the 24 7-branes are commonly referred to as F-theory 7-branes [26].

Close to $\omega=0 j$ has a pole, which must correspond to $q=0$, i.e. $\operatorname{Im} \lambda \sim \infty$, in the left-hand side of (3.27). Near $q=0 j(\lambda)$ takes the form

$$
j(\lambda) \sim e^{-2 \pi i \lambda},
$$

so that we can determine the precise form of $\lambda(\omega)$ :

$$
\lambda=\frac{1}{2 \pi i} \log \omega .
$$

This is the axion-dilaton modulus of the D7-brane solution (3.19).

In order to study the form of the solution at strong coupling we use that $j(-1 / \tau)=$ $j(\tau)$ and consider the limit $|\tau| \sim 0$, so that close to $\omega=0 j(\lambda)$ behaves as

$$
j(\lambda) \sim e^{2 \pi i / \lambda},
$$

for $\lambda \rightarrow 0$. Therefore, near $\omega=0$ we have

$$
\lambda=-\frac{2 \pi i}{\log \omega},
$$

which corresponds to the NS-7B brane solution (3.26). Therefore the D7 and NS-7B brane solutions are just the weak and strong coupling limits of the non-perturbative 7-brane solution of [21]. We will give further evidence in Section 6 for the fact that they represent the same physical object but in different coordinates patches, where changing the patch amounts to choosing a different region to parametrize the $S L(2, \mathbb{Z})$ moduli space.

From the point of view of the low energy effective actions we can conclude that the introduction of three 8-form potentials is necessary when we want to study the transformation of the D7-brane under $S L(2, \mathbb{Z})$, in particular when trying to map the $C^{(8)}$ field onto a local potential. The NS-7B brane effective action that we have constructed should be interpreted as the source for a 7-brane at strong coupling, where the $C^{(8)}$ field becomes $\tilde{C}^{(8)}$, which is non-local in terms of $C^{(8)}$ and $B^{(8)}$. Moreover, although $B^{(8)}$ has a meaning as the electric-magnetic dual of the dilaton, $\tilde{C}^{(8)}$ cannot be considered as an independent field in the Type IIB theory (contributing with one unit to the counting of 
the bosonic degrees of freedom) given that it is a function of the other 8-forms. This gives already an indication that the NS-7B brane should not have associated a central charge in the Type IIB space-time supersymmetry algebra. We give further evidence to this suggestion in Section 6, by showing that the two solutions are related by a coordinate transformation in the transverse space which is equivalent to changing the fundamental region of $S L(2, \mathbb{Z})$ by its $S$-dual. Similar observations will also apply for the KK-6A and KK-8A branes related to the NS-7B brane by T-duality.

\subsection{NS-7B branes and orientifold constructions}

There are particular limits of F-theory on K3 for which $\lambda$ remains constant over the base. In these limits $\mathrm{K} 3$ degenerates into a certain orbifold of $T^{4}$, which can be made equivalent to an orientifold of Type IIB on $T^{2}$. The most clear case is that of F-theory on $T^{4} / \mathbb{Z}_{2}$ [29]. Sen showed that in this branch F-theory is equivalent to Type IIB on $T^{2}$ modded out by the symmetry $(-1)^{F_{L}^{s}} \Omega I_{89}$, where $F_{L}^{s}$ is the space-time fermion number of the left-movers, $\Omega$ is the world-sheet parity reversal and $I_{89}$ is the space-time inversion in the two directions of the torus. In this Type IIB orientifold there are four fixed planes whose charge is cancelled by the addition of four D7-branes (plus their mirrors). Open strings ending on these 7-branes provide $S O(8)^{4}$ Chan-Paton factors, and this gauge structure coincides with the gauge symmetry of F-theory on $T^{4} / \mathbb{Z}_{2}$. Therefore in this particular branch it is possible to give a description of the gauge structure of the theory in terms of BPS states in weakly coupled Type IIB string theory.

The situation is not so simple in other branches, where the symmetry by which one has to mod out in the Type IIB description involves non-perturbative operations [30]. This is what happens in F-theory on $T^{4} / \mathbb{Z}_{4}$, realized as an orientifold of Type IIB on $T^{2}$ modded by the $\mathbb{Z}_{4}$ group generated by $S R^{(4)}$, where $S$ is the $\mathrm{S}$-duality generator and $R^{(4)}$ rotates $\pi / 2$ in the 8,9 plane. Another case is F-theory on $T^{4} / \mathbb{Z}_{6}$, which corresponds to Type IIB on $T^{2} / \mathbb{Z}_{6}$, where the group is generated by $(S T) R^{(6)}$, where $T$ is the T-generator of $S L(2, \mathbb{Z})$ and $R^{(6)}$ rotates $\pi / 3$ in the 8,9 plane. In these limits of F-theory the orientifold fixed planes in the Type IIB description are non-perturbative and they require the addition of non-perturbative 7-branes. The analysis of the singularities on the F-theory side reveals that on the $T^{4} / \mathbb{Z}_{4}$ branch the gauge structure is $E_{7} \times E_{7} \times S O(8)$, and in $T^{4} / \mathbb{Z}_{6}, E_{8} \times E_{6} \times S O(8)$.

The Type IIB BPS states responsible for the gauge symmetries of these branches have been identified in [31], as well as the way the $A_{n}$ gauge groups, associated to $n$ coinciding branes, are enhanced so as to recover the gauge group associated to the singularity structure (see also Refs. [32,33]). These BPS states correspond to three different types of non-perturbative 7-branes, denoted as $A, B, C$, such that for a $D_{n}$ singularity there are $A^{n} B C$ 7-branes located at the singularity, and $A^{n-1} B C^{2}$ for a $E_{n}$ singularity. At weak coupling the $B C$ 7-branes collapse to an orientifold plane $\mathrm{O}$ [34] and the gauge group that emerges is the full $S O(2 n)$ for a $D_{n}$ singularity and a subgroup $S O(2(n-1)) \times U(1)$ for a $E_{n}$, which can be associated to Chan-Paton factors of fundamental strings, given that the $A^{n}$ branes are D7-branes, located on top of the $\mathrm{O}$ fixed plane. Therefore for $D_{n}$ singularities the gauge structure is completely captured perturbatively. In the strong coupling limit the same reasoning applies, but now the $A^{n}$ branes are NS-7B branes, located on top of the S-dual orientifold fixed planes, and the 
Chan-Paton factors are associated to open D-strings. At intermediate couplings the gauge groups are enhanced, essentially because it is necessary to consider different sheets to describe the 7-branes, and then the same 7-brane can be seen as a different brane in another sheet, so that there can be open strings connecting them through complicated paths [31] (see Refs. [32,33] for a description in terms of string junctions).

\section{The IIA KK8-brane}

The NS-7B brane predicts the existence of a new brane in Type IIA which can be obtained from it by T-duality. This brane is also related to the M9-brane as follows [8]. The effective action of a single M9-brane contains a gauged direction in its world-volume $[8,11]$ in such a way that the field content is that of the nine-dimensional vector multiplet. Reduction along this gauged direction gives the D8-brane effective action, whereas the reduction along the transverse direction gives the action of the NS-9A brane. There is a third possibility, namely the reduction along a world-volume direction other than the Killing direction. This gives an 8-brane with a gauged direction in its world-volume, inherited from the M9-brane, which we denote as a KK-8A brane. In the notation of [8] it corresponds to the $\left(7,1^{3} ; 3\right)$ brane predicted by U-duality of M-theory on $T^{8}$. The presence of this kind of 8-branes in the background is the origin of the mass of the massive Type IIA supergravity that is obtained by reducing the massive 11-dimensional supergravity of [35] along a direction different from the Killing direction.

As explained in [35] it is necessary to introduce a Killing direction in order to formulate a massive supergravity in 11 dimensions. The theory is therefore not fully covariant and this is the way the no-go theorem of [36] is circumvented. The origin of the mass is the presence of M9-branes in the background, which couple as well to the Killing vector in their world-volumes. Romans massive supergravity [37] is obtained by reducing along the Killing direction, therefore is fully covariant, with the presence of D8-branes in the background being the origin of the mass [38]. On the other hand, the reduction along a direction different from the Killing direction gives rise to a non-covariant Type IIA supergravity, containing a Killing vector. The presence of KK-8A branes in the background is the origin of the mass of this theory.

Massive Type II nine-dimensional supergravity is obtained by reducing Romans massive supergravity to nine dimensions, being the presence of D7-branes in the background the origin of its mass [38]. If instead one reduces the massive Type IIA supergravity with Killing direction and KK-8A branes along the Killing direction, one gets a massive nine-dimensional supergravity in which the mass is induced by the presence of NS-7B branes, T-dual to the KK-8A branes. This theory is S-dual to the massive nine-dimensional supergravity coming from the reduction of Romans massive IIA supergravity, given that from the 11-dimensional point of view one is just interchanging the two compactified directions. In order to obtain a manifestly $S L(2, \mathbb{R})$ invariant massive nine-dimensional supergravity, in which both D7 and NS-7B branes are present in the background, it is necessary to perform a generalized dimensional reduction, as explained in [13].

One way to construct the action of the KK-8A brane is by dimensional reduction from the M9-brane. However, only the kinetic term of the M9-brane effective action has 
been constructed in the literature $[11,12]$. Therefore, in this section we derive the KK-8A brane effective action by performing a T-duality transformation in the NS-7B brane. The T-duality takes place along a transverse direction, which in the dual KK-8A brane plays the role of Killing direction. We interpret this direction as world-volume because it is the one inherited from the M9-brane in the double-dimensional reduction explained before.

The result is the following effective action:

$$
\begin{aligned}
S_{\mathrm{KK} 8 \mathrm{~A}}= & -T_{\mathrm{KK} 8 \mathrm{~A}} \int d^{8} \xi e^{-3 \phi}|k|^{3}\left(1+e^{2 \phi}|k|^{-2}\left(i_{k} C^{(1)}\right)^{2}\right) \\
& \times\left\{\mid \operatorname{det}\left(D_{i} X^{\mu} D_{j} X^{\nu} g_{\mu \nu}-\left(2 \pi \alpha^{\prime}\right)^{2}|k|^{-2} \mathscr{K}_{i}^{(1)} \mathscr{K}_{j}^{(1)}\right.\right. \\
& \left.\left.+\frac{\left(2 \pi \alpha^{\prime}\right)|k|^{-1} e^{\phi}}{\sqrt{1+e^{2 \phi}|k|^{-2}\left(i_{k} C^{(1)}\right)^{2}}} \mathscr{K}_{i j}^{(2)}\right) \mid\right\}^{1 / 2} \\
& +\frac{1}{8 !}\left(2 \pi \alpha^{\prime}\right) T_{\mathrm{KK} 8 \mathrm{~A}} \int d^{8} \xi \epsilon^{i_{1} \ldots i_{8}} \mathscr{K}_{i_{1} \ldots i_{8}}^{(8)} .
\end{aligned}
$$

The kinetic term is very similar to that of the Type IIA KK-monopole (see Ref. [23]), although now the factors in front of the square root have different powers. The covariant derivative is the usual one for KK-monopoles,

$$
D X^{\mu}=\partial X^{\mu}+A k^{\mu},
$$

where $k^{\mu} \partial / \partial X^{\mu}=\partial / \partial z$ and $z$ is the direction along which we T-dualized the NS-7B brane. Therefore this direction becomes a Killing direction after T-duality. The dependent gauge field is given, as usual, by $A=|k|^{-2} \partial X^{\mu} k_{\mu}$. The field strengths $\mathscr{K}^{(1)}$ and $\mathscr{K}^{(2)}$ have the form

$$
\begin{aligned}
& \mathscr{K}^{(1)}=\partial \omega^{(0)}-\frac{1}{2 \pi \alpha^{\prime}}\left(i_{k} B^{(2)}\right), \\
& \mathscr{K}^{(2)}=2 \partial \omega^{(1)}+\frac{1}{2 \pi \alpha^{\prime}}\left(i_{k} C^{(3)}\right)-2 \mathscr{K}^{(1)}\left(D X C^{(1)}\right),
\end{aligned}
$$

where $\omega^{(0)}$ and $\omega^{(1)}$ arise from the T-duality transformations ${ }^{10}$

$$
c^{(1)^{\prime}}=-\omega^{(1)}, \quad z^{\prime}=\left(2 \pi \alpha^{\prime}\right) \omega^{(0)},
$$

and we also have

$$
\tilde{\mathscr{F}}^{\prime}=-\mathscr{K}^{(2)} \text {. }
$$

\footnotetext{
${ }^{10}$ Note that $\omega^{(1)}$ and $d^{(1)}$ (in (2.9)) have different gauge transformations.
} 
$\tilde{\omega}^{(7)}$ is T-dual to $\tilde{c}^{(7)}$ in (3.2) and plays the role of tension of the KK-8A brane. Finally, the WZ term reads

$$
\begin{aligned}
& \mathscr{K}^{(8)}=8\left\{\partial \tilde{\omega}^{(7)}-\frac{1}{8\left(2 \pi \alpha^{\prime}\right)}\left(i_{k} N^{(9)}\right)-\left(i_{k} N^{(8)}\right) \partial \omega^{(0)}\right. \\
& +\frac{7}{2}\left(i_{k} N^{(7)}\right)\left[2 \partial \omega^{(1)}+\frac{1}{2 \pi \alpha^{\prime}}\left(i_{k} C^{(3)}\right)+\frac{2}{2 \pi \alpha^{\prime}}\left(i_{k} B^{(2)}\right)\left(D X C^{(1)}\right)\right] \\
& -21\left(2 \pi \alpha^{\prime}\right)\left(i_{k} B^{(6)}\right) \mathscr{K}^{(1)} \mathscr{K}^{(2)} \\
& -\frac{105}{4}\left(2 \pi \alpha^{\prime}\right)\left(i_{k} C^{(5)}\right)\left[\mathscr{K}^{(2)}-4\left(D X C^{(1)}\right) \mathscr{K}^{(1)}\right] \mathscr{K}^{(2)} \\
& +70 D X^{\mu_{1}} D X^{\mu_{2}} D X^{\mu_{3}} C_{\mu_{1} \mu_{2} \mu_{3}}^{(3)}\left[\left(i_{k} C^{(3)}\right)\left(i_{k} B^{(2)}\right)\right. \\
& \left.-3\left(2 \pi \alpha^{\prime}\right)^{2} \mathscr{K}^{(1)} \partial \omega^{(1)}\right] \mathscr{K}^{(2)} \\
& -35 D X^{\mu_{1}} D X^{\mu_{2}} D X^{\mu_{3}} C_{\mu_{1} \mu_{2} \mu_{3}}^{(3)}\left(i_{k} C^{(3)}\right)^{2} \partial \omega^{(0)} \\
& -70\left[2 C^{(3)}\left(i_{k} B^{(2)}\right)+3\left(i_{k} C^{(3)}\right) B^{(2)}\right]\left(i_{k} C^{(3)}\right)\left(D X C^{(1)}\right) \partial \omega^{(0)} \\
& +105 D X^{\mu_{1}} D X^{\mu_{2}} B_{\mu_{1} \mu_{2}}^{(2)}\left[\left(i_{k} C^{(3)}\right)^{2} \mathscr{K}^{(2)}-4\left(2 \pi \alpha^{\prime}\right)^{2}\left(\partial \omega^{(1)}\right)^{3}\right] \\
& -\frac{7 !}{3 !}\left(2 \pi \alpha^{\prime}\right)^{3} A \partial \omega^{(0)}\left(\partial \omega^{(1)}\right)^{3} \\
& \left.+\frac{7 !}{3 !}\left(2 \pi \alpha^{\prime}\right)^{3} \frac{e^{2 \phi} k^{-2}\left(i_{k} C^{(1)}\right)}{1+e^{2 \phi} k^{-2}\left(i_{k} C^{(1)}\right)^{2}}\left(\mathscr{K}^{(2)}\right)^{4}\right\} .
\end{aligned}
$$

This Wess-Zumino 8-form comes from (3.2) after T-duality along the transverse direction $z$. The T-duality transformation rule for the field $\tilde{C}^{(8)}$ is

$$
\begin{aligned}
\tilde{C}_{\mu_{1} \ldots \mu_{7} z}^{(8)^{\prime}}= & \left(i_{k} N^{(8)}\right)_{\mu_{1} \ldots \mu_{7}}+7\left(i_{k} N^{(7)}\right)_{\left[\mu_{1} \ldots \mu_{6}\right.}\left(C_{\left.\mu_{7}\right]}^{(1)}-C_{z}^{(1)} \frac{g_{\left.z \mu_{7}\right]}}{g_{z z}}\right) \\
& +35\left(C_{\left[\mu_{1} \ldots \mu_{3}\right.}^{(3)}-3 C_{\left[\mu_{1} \mu_{2} z\right.}^{(3)} \frac{g_{z \mu_{3}}}{g_{z z}}\right) C_{\mu_{4} \mu_{5} z}^{(3)} C_{\left.\mu_{6} \mu_{7}\right] z}^{(3)} \\
& +70\left[2 C_{\left[\mu_{1} \ldots \mu_{3}\right.}^{(3)} B_{\mu_{4} z}^{(2)}+3 C_{\left[\mu_{1} \mu_{2} z\right.}^{(3)} B_{\mu_{3} \mu_{4}}^{(2)}\right] C_{\mu_{5} \mu_{6} z}^{(3)}\left(C_{\left.\mu_{7}\right]}^{(1)}-C_{z}^{(1)} \frac{g_{\left.z \mu_{7}\right]}}{g_{z z}}\right),
\end{aligned}
$$

where $i_{k} N^{(8)}$ is the gravitational field to which the KK-6A brane couples minimally (see (2.12)), and

$$
\tilde{C}_{\mu_{1} \ldots \mu_{8}}^{(8)^{\prime}}=\left(i_{k} N^{(9)}\right)_{\mu_{1} \ldots \mu_{8}} .
$$

This last relation defines a new field $N^{(9)}$ (its gauge transformation rule can be found in Appendix A), which is electric-magnetic dual to the mass parameter of the massive Type IIA supergravity that is obtained by reducing the massive 11-dimensional supergravity of Ref. [35] along a direction different from the Killing direction (see Section 7). 
As we have seen, this Killing direction is also present in the world-volume of the KK-8A brane. The field content of the KK-8A brane is that of the eight-dimensional vector multiplet, containing one vector field: $\omega^{(1)}$, and two scalars: $\omega^{(0)}$ and the transverse embedding scalar.

To finish this section let us analyze the soliton solutions in the world-volume of the KK-8A brane. These can be derived by T-duality from the configurations involving the NS-7B brane, and also from the configurations associated to the M9-brane given in $[39,40]$ :

- $\quad$ The 1-form $\omega^{(1)}$ couples to a 0-brane soliton originated by the configuration $(0 \mid \mathrm{D} 2, \mathrm{KK} 8 \mathrm{~A})$. This is related by T-duality to $(0 \mid \mathrm{D} 1, \mathrm{NS} 7 \mathrm{~B})$ and by reduction to (1|M2,M9) [39,40]. This 1-brane soliton is however realized as a 0-brane soliton in the effective nine-dimensional world-volume of the M9-brane, given that one of the world-volume directions of the M2-brane is wrapped around the isometric direction. Reduction along an ordinary world-volume direction of the M9-brane gives rise to $(0 \mid \mathrm{D} 2, \mathrm{KK} 8 \mathrm{~A}) . \omega^{(1)}$ also couples magnetically to a 4-brane soliton, associated to the configurations (4|NS5A,KK8A) and (4|D4,KK8A). The configuration (4|NS5A,KK8A) is related by T-duality to (4|NS5B,NS7B) and is obtained from the configuration (5|M5,M9), where the M5-brane is wrapped around the Killing direction of the M9-brane. Therefore this soliton is realized as a 4-brane soliton in the nine-dimensional world-volume of the M9-brane. On the other hand, the configuration (4|D4,KK8A) is related by T-duality to (4|D5,NS7B) and it is also obtained from (5|M5,M9), with the M5 embedded in the nine-dimensional world-volume of the M9-brane and the reduction taking place along a common world-volume direction.

- $\quad$ The 0 -form $\omega^{(0)}$ couples magnetically to the 5-brane soliton originated by the configuration (5|NS5A,KK8A), where the NS-5A brane is embedded in the KK-8A brane. This configuration also arises from the reduction of (5|M5,M9). It is related by T-duality to (5|KK5B,NS7B).

- The only physical embedding scalar can be associated to the boundary of a KK-6A brane, where we make coincide the isometries of both, the KK-6A and the KK-8A branes: (5|KK6A,KK8A). This configuration arises as the dimensional reduction of $(5 \mid \mathrm{MKK}, \mathrm{M} 9)$ along a direction of the world-volume of the M9-brane transversal to the MKK-monopole.

- $\quad$ Since the KK-8A brane has the interpretation of an 8-brane in ten dimensions, it is a domain wall, hence it is not expected to have a boundary. Thus we do not associate any physical meaning to the 7 -form $\tilde{\omega}^{(7)}$.

\section{The IIB KK7-brane}

In this section we present the explicit effective action of an exotic brane with two Killing isometries. This brane is interpreted as a 7-brane in the Type IIB theory, and is non-perturbative in the same sense as the branes that we have discussed previously, since its tension also scales like $e^{-3 \phi}$. It is related to the KK-6A brane by T-duality along a transverse direction and to the KK-8A brane by T-duality along a world-volume direction. This last connection allows us to interpret this brane as the one responsible for the mass of a massive nine-dimensional Type II supergravity containing a Killing 
direction. This theory should give rise to an eight-dimensional massive supergravity with no Killing directions.

We construct the world-volume effective action by performing a T-duality transformation in the KK-6A brane along a transverse coordinate. The derivation starting from the KK-8A brane will also be discussed at the end of the section. The result of these duality transformations is a brane with 6 ordinary spatial directions and two gauged isometries $[8,13]$, which in the notation of [8] corresponds to the $\left(6,1^{2}, 1^{3} ; 3\right)$ exotic brane predicted by $\mathrm{U}$-duality of M-theory on an 8-torus. It has an effective tension

$$
\mathscr{T}_{\text {KK } 7 \mathrm{~B}}=\frac{R_{1}^{2} R_{2}^{3}}{(2 \pi)^{6} g_{s}^{3} l_{s}^{12}},
$$

where $R_{1}, R_{2}$ are the radii of the two Killing directions. We call this brane a KK-7B brane, since tracing back the origin of its two Killing directions we find that one has its origin in the isometry of the MKK monopole, which is interpreted as a transverse direction, whereas the second Killing direction has its origin in the isometry of the M9-brane, which is associated to a world-volume direction. Therefore, although this brane contains only six ordinary spatial directions, it is in fact interpreted as a 7-brane.

Since the exact world-volume effective action is rather complicated we have considered the case $B^{(2)}=C^{(2)}=0$. Starting with the effective action of the KK-6A brane we perform a T-duality transformation along a transverse direction different from its Taub-NUT direction. This direction becomes a Killing direction for the dual brane. Denoting $h^{\mu}$ the Killing vector associated to the new isometry the kinetic term in the dual effective action is given by

$$
\begin{aligned}
& S_{\text {КК 7B }} \\
& =-T_{\text {КK 7B }} \int d^{7} \xi e^{-3 \phi}|h|\left|k^{2} h^{2}-(k \cdot h)^{2}\right| \\
& \quad \times\left\{\mid \operatorname{det}\left(D_{i} X^{\mu} D_{j} X^{\nu} g_{\mu \nu}-\left(2 \pi \alpha^{\prime}\right)^{2}|h|^{-2} e^{\phi} \mathscr{K}_{i}^{T} M \mathscr{K}_{j}\right.\right. \\
& \left.\left.\quad+\left(2 \pi \alpha^{\prime}\right)\left|k^{2} h^{2}-(k \cdot h)^{2}\right|^{-1 / 2} e^{\phi} \mathscr{G}_{i j}^{(2)}\right) \mid\right\}^{1 / 2} .
\end{aligned}
$$

Here the covariant derivatives contain the two Killing vectors $k^{\mu}$ and $h^{\mu}$,

$$
D X^{\mu}=\partial X^{\mu}+A^{(1)} k^{\mu}+A^{(2)} h^{\mu},
$$

and the two dependent gauge vector fields $A^{(1)}, A^{(2)}$ are defined as ${ }^{11}$

$$
A_{i}^{(1)}=\frac{h^{2} k_{i}-(k . h) h_{i}}{k^{2} h^{2}-(k . h)^{2}}, \quad A_{i}^{(2)}=\frac{k^{2} h_{i}-(k \cdot h) k_{i}}{k^{2} h^{2}-(k \cdot h)^{2}} .
$$

$\mathscr{K}^{T} M \mathscr{K}$ is the $\operatorname{SL}(2, \mathbb{R})$-invariant combination ${ }^{12}$

$$
\mathscr{K}^{T} M \mathscr{K}=\left(\begin{array}{ll}
\mathscr{R}^{(1)} & \tilde{\mathscr{K}}^{(1)}
\end{array}\right) e^{\phi}\left(\begin{array}{cc}
e^{-2 \phi}+C^{(0) 2} & C^{(0)} \\
C^{(0)} & 1
\end{array}\right)\left(\begin{array}{c}
\mathscr{K}^{(1)} \\
\tilde{\mathscr{K}}^{(1)}
\end{array}\right),
$$

\footnotetext{
${ }^{11}$ In our notation, $(k \cdot h)=-g_{\mu \nu} k^{\mu} h^{\nu}$.

12 This combination appeared already in the effective action of the Type IIB KK-monopole [17], which behaves as a singlet under S-duality transformations.
} 
with

$$
\mathscr{K}^{(1)}=\partial \omega^{(0)}, \quad \tilde{\mathscr{K}}^{(1)}=\partial \tilde{\omega}^{(0)} .
$$

The two scalar fields $\omega^{(0)}, \widetilde{\omega}^{(0)}$ transform as a doublet under S-duality:

$$
\omega^{(0)} \rightarrow \tilde{\omega}^{(0)}, \quad \tilde{\omega}^{(0)} \rightarrow-\omega^{(0)},
$$

and arise in the T-duality transformation as

$$
y^{\prime}=\left(2 \pi \alpha^{\prime}\right) \omega^{(0)}, \quad c^{(0)^{\prime}}=-\widetilde{\omega}^{(0)} .
$$

Note that here $y$ denotes the direction along which the T-duality takes place, and should not be mistaken with the eleventh direction in Section 2. Within M-theory, recalling that $c^{(0)}$ (in the effective action of the KK-6A brane) is related through rescaling to the eleventh direction, we clearly see that the S-duality transformation of the two scalars $\omega^{(0)}, \tilde{\omega}^{(0)}$ is induced from a modular transformation in the 2-torus.

$\mathscr{G}^{(2)}$ is given by

$$
\mathscr{G}^{(2)}=2 \partial d^{(1)}+\frac{1}{2 \pi \alpha^{\prime}}\left(i_{h} i_{k} C^{(4)}\right)+2\left(2 \pi \alpha^{\prime}\right) \frac{(k \cdot h)}{h^{2}} \partial \omega^{(0)} \partial \tilde{\omega}^{(0)},
$$

and arises after T-duality as

$$
\mathscr{H}^{(2)^{\prime}}=\mathscr{G}^{(2)} \text {. }
$$

The Wess-Zumino term reads

$$
\begin{aligned}
S_{\mathrm{KК} 7 \mathrm{~B}}^{\mathrm{WZ}}= & \frac{1}{6 !}\left(2 \pi \alpha^{\prime}\right) T_{\text {КК } 7 \mathrm{~B}} \int d d^{7} \xi \epsilon \partial \tilde{\omega}^{(6)}+\frac{1}{7\left(2 \pi \alpha^{\prime}\right)}\left(i_{h} i_{k} N^{(9)}\right)+\left(i_{h} i_{k} N^{(8)}\right) \partial \omega^{(0)} \\
& +\left(i_{h} i_{k} \mathscr{N}^{(8)}\right) \partial \tilde{\omega}^{(0)}-6\left(2 \pi \alpha^{\prime}\right)\left(i_{k} i_{h} N^{(7)}\right) \partial \omega^{(0)} \partial \tilde{\omega}^{(0)}-6\left(i_{k} i_{h} \mathscr{N}^{(7)}\right) \partial d^{(1)} \\
& +5 D X^{\mu} D X^{\nu} D X^{\rho}\left(i_{h} C^{(4)}\right)_{\mu \nu \rho}\left[\frac{1}{2 \pi \alpha^{\prime}}\left(i_{h} i_{k} C^{(4)}\right)^{2}\right. \\
& \left.+6\left(2 \pi \alpha^{\prime}\right) \partial d^{(1)}\left(2 \partial d^{(1)}+\frac{1}{2 \pi \alpha^{\prime}}\left(i_{h} i_{k} C^{(4)}\right)\right)\right] \\
& +120\left(2 \pi \alpha^{\prime}\right)^{2} D X^{\mu} D X^{\nu} D X^{\rho}\left(i_{k} C^{(4)}\right)_{\mu \nu \rho} \partial d^{(1)} \partial \omega^{(0)} \partial \tilde{\omega}^{(0)} \\
& +30\left(2 \pi \alpha^{\prime}\right) D X^{\mu} D X^{\nu} D X^{\rho}\left(i_{k} C^{(4)}\right)_{\mu \nu \rho}\left(i_{h} i_{k} C^{(4)}\right) \partial \omega^{(0)} \partial \tilde{\omega}^{(0)} \\
& \left.+360\left(2 \pi \alpha^{\prime}\right)^{3}\left(\partial d^{(1)}\right)^{2} \partial \omega^{(0)} \partial \tilde{\omega}^{(0)} A^{(2)}-120\left(2 \pi \alpha^{\prime}\right)^{2} A^{(1)}\left(\partial d^{(1)}\right)^{3}\right\} .
\end{aligned}
$$

The new fields $N^{(9)}, N^{(8)}, \mathscr{N}^{(8)}$, arise from the T-duality of the fields $N^{(8)}, N^{(7)}$ in the Type IIA theory. Namely, in our truncation

$$
\begin{aligned}
& \left(i_{k} N^{(8)^{\prime}}\right)_{\mu_{1} \ldots \mu_{7}}=\left(i_{k} i_{h} N^{(9)}\right)_{\mu_{1} \ldots \mu_{7}}+21\left(i_{k} i_{h} \mathscr{N}^{(7)}\right)_{\left[\mu_{1} \ldots \mu_{5}\right.}\left(i_{k} i_{h} C^{(4)}\right)_{\left.\mu_{6} \mu_{7}\right]}, \\
& \left(i_{k} N^{(8)^{\prime}}\right)_{\mu_{1} \ldots \mu_{6} y}=\left(i_{h} i_{k} N^{(8)}\right)_{\mu_{1} \ldots \mu_{6}}, \\
& \left(i_{k} N^{(7)^{\prime}}\right)_{\mu_{1} \ldots \mu_{6}}=\left(i_{h} i_{k} \mathscr{N}^{(8)}\right)_{\mu_{1} \ldots \mu_{6}} .
\end{aligned}
$$

Their gauge transformation rules can be found in Appendix A. 
Similarly,

$$
\begin{aligned}
\left(i_{k} N^{(7)^{\prime}}\right)_{\mu_{1} \ldots \mu_{5} y}= & \left(i_{k} i_{h} N^{(7)}\right)_{\mu_{1} \ldots \mu_{5}}-\left(i_{k} i_{h} \mathscr{N}^{(7)}\right)_{\mu_{1} \ldots \mu_{5}} \frac{g_{y z}}{g_{y y}} \\
& -5\left(i_{k} C^{(4)}\right)_{\left[\mu_{1} \ldots \mu_{3}\right.}\left(i_{k} i_{h} C^{(4)}\right)_{\left.\mu_{4} \mu_{5}\right]}-5\left(i_{k} i_{h} C^{(4)}\right)_{\left[\mu_{1} \ldots \mu_{4}\right.}^{2} \frac{g_{\left.\mu_{5}\right] y}}{g_{y y}} \\
& +\frac{5}{3}\left(i_{h} C^{(4)}\right)_{\left[\mu_{1} \mu_{2} \mu_{3}\right.}\left(i_{k} i_{h} C^{(4)}\right)_{\left.\mu_{4} \mu_{5}\right]} \frac{g_{y z}}{g_{y y}}
\end{aligned}
$$

again in the same truncation. The field $N^{(7)}$ in the Type IIB theory is the electric-magnetic dual of the Killing vector $k_{\mu}$ considered as a 1-form. This field is the one to which the Type IIB Kaluza-Klein monopole couples minimally, when $k^{\mu}$ is taken along the Taub-NUT direction. Its gauge transformation rule can be found in [17]. In the action of the KK-7B brane, with two Killing directions, there is another 7-form field, $\mathscr{N}^{(7)}$, electric-magnetic dual of the second Killing vector, $h_{\mu}$. Analogously $N^{(8)}$ and $\mathscr{N}^{(8)}$ must be the electric-magnetic duals of the two scalars $k^{2}, h^{2}$. The field $N^{(9)}$ is dual to a mass parameter, which should be the one of the nine-dimensional Type II massive supergravity with a Killing isometry to which this 7-brane gives mass. $\tilde{\omega}^{(6)}$ is T-dual to $\omega^{(6)}$ in (2.12) and plays the role of tension of the Type IIB KK7-brane.

The effective action of the KK-7B brane is manifestly invariant under the two local isometric transformations generated by $k^{\mu}$ and $h^{\mu}$,

$$
\delta X^{\mu}=-\sigma^{(1)}(\xi) k^{\mu}-\sigma^{(2)}(\xi) h^{\mu} .
$$

It is also easy to check that it is S-duality invariant. The fields $N^{(9)}, N^{(7)}$ and $\mathscr{N}^{(7)}$ are S-self-dual whereas $N^{(8)}, \mathscr{N}^{(8)}$ transform as a doublet. This can be deduced from their origin in 11 dimensions, where $\mathrm{S}$-duality is realized as a modular transformation in $T^{2}$.

As we have mentioned before the KK-7B brane effective action can be derived as well from the KK-8A brane, by performing a T-duality transformation along a worldvolume direction. In this case the T-duality mapping of the field strengths goes as follows:

$$
\begin{aligned}
& \mathscr{K}_{i j}^{(2)^{\prime}}-2\left(2 \pi \alpha^{\prime}\right) \frac{(k \cdot h)}{k^{2}} \mathscr{K}_{[i}^{(1)^{\prime}} \mathscr{K}_{j] \sigma}^{(2)^{\prime}}=-\mathscr{G}_{i j}^{(2)}, \\
& \mathscr{K}_{i}^{(1)^{\prime}} \mathscr{K}_{j}^{(1)^{\prime}}+e^{2 \phi} \mathscr{K}_{i \sigma}^{(2)^{\prime}} \mathscr{K}_{j \sigma}^{(2)^{\prime}}=e^{\phi} \mathscr{K}_{i}^{T} M \mathscr{K}_{j} .
\end{aligned}
$$

This connection with the KK-8A brane shows that one of the two Killing directions should be interpreted as a world-volume direction, given that from this calculation it is inherited from the world-volume isometry of the KK-8A brane.

The world-volume fields present in the effective action describe soliton solutions in the world-volume of the brane. In this case, with two gauged isometries, the field strengths are of the form

$$
\mathscr{K}^{(p-1)}=(p-1) \partial \omega^{(p-2)}+\frac{1}{2 \pi \alpha^{\prime}}\left(i_{h} i_{k} C^{(p+1)}\right)+\ldots
$$


Therefore a $(p-2)$-form in the world-volume couples to a $(p-3)$-brane soliton realized as the boundary of a $p$-brane wrapped around the two compact directions. We find the following soliton solutions in the world-volume of the KK-7B brane:

- $\quad$ The 1-form $d^{(1)}$ couples to a 0-brane soliton originated by a D3-brane with two of its world-volume directions wrapped around the Killing directions of the KK-7B brane: (0|D3,KK7B). This configuration is a singlet under S-duality and is related by T-duality to $(0 \mid \mathrm{D} 2, \mathrm{KK} 6 \mathrm{~A})$. A 0 -brane soliton can also originate by a $(p, q)$ string wrapped around one of the Killing directions of the KK7B. The $(0 \mid \mathrm{D} 1, \mathrm{KK} 7 \mathrm{~B})$ configuration is related by T-duality to $(0 \mid \mathrm{D} 2, \mathrm{KK} 6 \mathrm{~A})$, whereas the $(0 \mid \mathrm{F} 1, \mathrm{KK} 7 \mathrm{~B})$ is related to $(0 \mid \mathrm{F} 1, \mathrm{KK} 6 \mathrm{~A}) . d^{(1)}$ also couples magnetically to a 3-brane soliton, realized as a $(p, q)$ 5-brane wrapped around the two Killing directions of the KK7B-brane. These configurations are T-dual to (3|NS5A,KK6A) and (3|D4,KK6A).

- $\quad$ The field strengths of the two scalars $\omega^{(0)}$ and $\tilde{\omega}^{(0)}$ involve the projection with the Killing vectors of the 2-forms $C^{(2)}$ and $B^{(2)}$, which we have not included. Accordingly, the doublet $\left(\omega^{(0)}, \tilde{\omega}^{(0)}\right)$ will couple to the four-dimensional boundary of a $(p, q)$ 5-brane which is wrapped around one of the Killing directions. T-duality relates these configurations with (4|D6,KK6A) and (4|KK5A,KK6A).

- $\quad$ The embedding scalars are, as usual, associated to the boundary of other Type II KK $p$ branes. However, the branes involved in this case are some of the exotic branes that we have not considered in this paper (see Section 7), so we will omit the explicit configurations.

- $\quad$ We find as well a domain-wall type of soliton, realized as $(5 \mid \mathrm{KK} 5 \mathrm{~B}, \mathrm{KK} 7 \mathrm{~B})$, coupled to $\tilde{\omega}^{(6)}$, and T-dual to (5|NS5A,KK6A).

\section{M-theory interpretation}

In this section we study the KK6A and NS7B branes from the point of view of the M-theory Kaluza-Klein monopole solution, from which they can be derived through reduction and duality ${ }^{13}$. We are able to show that if one imposes an extra isometry in this solution then it can be written as a 2-torus bundle over a two-dimensional transverse space and that a modular transformation in the torus can be undone by a change of coordinates in the transverse space. This symmetry allows us to connect the KK6A with the D6 brane and the NS7B with the D7 brane. We discuss the consequences regarding the appearance of these branes in the space-time supersymmetry algebras.

Our starting point is the MKK-monopole solution, which arises when at least one direction in space-time is compact,

$$
d s_{\mathrm{MKK}}^{2}=d s_{6,1}^{2}-\frac{1}{H}(d z+\boldsymbol{V} \cdot d \boldsymbol{x})^{2}-H(d \boldsymbol{x} \cdot d \boldsymbol{x}) .
$$

\footnotetext{
${ }^{13}$ We thank Chris Hull, who has also considered 7-brane solutions and their relations to M-theory, for a conversation on this point.
} 
It represents a 6-brane in a four-dimensional transverse space, where $z$ is a Taub-NUT isometry direction and the function $H$ is harmonic in the three-dimensional space $\boldsymbol{x}=\left(x_{1}, x_{2}, x_{3}\right)$ and is related to $\boldsymbol{V}$ by $\boldsymbol{\nabla} H=\boldsymbol{\nabla} \wedge \boldsymbol{V}$. This solution is magnetically charged under the Kaluza-Klein vector $i_{k} g$, where $k$ is the Killing vector associated to the isometry direction $z$ and $g$ denotes the space-time metric.

If we impose an additional isometry in the transverse space of the monopole ${ }^{14}$ the harmonic function depends only on two of the three transversal coordinates, and we can gauge away two of the three components of the gauge vector $\boldsymbol{V}$. We make the choice such that $H=H\left(x_{1}, x_{2}\right)$ and $\boldsymbol{V}=\left(0,0, V_{3}\right)$, with $V_{3}=V_{3}\left(x_{1}, x_{2}\right)$. The monopole solution takes the form

$$
d s_{\mathrm{MKK}}^{2}=d s_{6,1}^{2}-\frac{1}{H}\left|d z+\left(V_{3}+i H\right) d x_{3}\right|^{2}-H\left(d x_{1}^{2}+d x_{2}^{2}\right) .
$$

This metric can be interpreted as a torus-bundle over a two-dimensional base space:

$$
d s_{\mathrm{MKK}}^{2}=d s_{6,1}^{2}-\frac{A}{\tau_{2}}\left|d z+\tau\left(x_{1}, x_{2}\right) d x_{3}\right|^{2}-R^{2}\left(d x_{1}^{2}+d x_{2}^{2}\right),
$$

where the fiber is a 2-torus with real periodic coordinates $\left(z, x_{3}\right) \sim\left(z+1, x_{3}+1\right)$, a constant area modulus $A$, which can be introduced by rescaling $z$ and $x_{3}$, and a complex structure $\tau=\tau_{1}+i \tau_{2}=V_{3}+i H$. This defines $\tau$ as a holomorphic function on the base space $x_{1}+i x_{2}$, or anti-holomorphic in $x_{2}+i x_{1}$. Finally, $R^{2}=H$ is the scale parameter of the base space. Therefore the MKK-monopole solution can be written as the product of two spaces $\mathbb{R}^{6,1} \times B$, where $B$ is the torus-bundle.

The fibers have an $S L(2, \mathbb{Z})$ symmetry,

$$
\tau \rightarrow \frac{a \tau+b}{c \tau+d}, \quad\left(\begin{array}{c}
z \\
x_{3}
\end{array}\right) \rightarrow\left(\begin{array}{cc}
a & -b \\
-c & d
\end{array}\right)\left(\begin{array}{c}
z \\
x_{3}
\end{array}\right) .
$$

Notice that $z$ and $x_{3}$ are now both isometry directions, $z$ is the Taub-NUT coordinate for a modulus $\tau$ and $x_{3}$ for a modulus $-\tau^{-1}$.

The solution (6.3), upon reduction over the torus fiber, has two wrapping modes, the D6-brane and the KK-6A brane. The D6-brane can be obtained by reducing over the coordinate $z$ (re-absorbing $A$ ),

$$
\begin{aligned}
& d s_{\mathrm{D} 6}^{2}=\tau_{2}^{-1 / 2} d s_{6,1}^{2}-R^{2} \tau_{2}^{-1 / 2}\left(d x_{1}^{2}+d x_{2}^{2}\right)-\tau_{2}^{1 / 2} d x_{3}^{2}, \\
& e^{\phi}=\tau_{2}^{-3 / 4}, \quad C^{(1)}=\tau_{1} d x_{3} .
\end{aligned}
$$

On the other hand, the KK-6A brane is defined as the reduction over the coordinate $x_{3}$. Taking $z$ along the M-theory circle, the KK-6A brane solution is given by [13]

$$
\begin{aligned}
& d s_{\mathrm{KK} 6 \mathrm{~A}}^{2}=\left(\frac{\tau_{2}}{|\tau|^{2}}\right)^{-1 / 2} d s_{6,1}^{2}-R^{2}\left(\frac{\tau_{2}}{|\tau|^{2}}\right)^{-1 / 2}\left(d x_{1}^{2}+d x_{2}^{2}\right)-\left(\frac{\tau_{2}}{|\tau|^{2}}\right)^{1 / 2} d x_{3}^{2}, \\
& e^{\phi}=\left(\frac{\tau_{2}}{|\tau|^{2}}\right)^{-3 / 4}, \quad C^{(1)}=-\frac{\tau_{1}}{|\tau|^{2}} d x_{3},
\end{aligned}
$$

\footnotetext{
${ }^{14}$ This can be achieved by considering an infinite array of monopoles [7].
} 
and is such that it is related to the D6-brane solution (6.5) by the transformation of the fiber modulus:

$$
\tau \rightarrow-\frac{1}{\tau}
$$

The coordinate transformation in the base space $\left(x_{1}, x_{2}\right) \rightarrow\left(y_{1}, y_{2}\right)$, where [24]

$$
d\left(y_{2}+i y_{1}\right)=\tau d\left(x_{2}+i x_{1}\right)
$$

yields $\tau_{2} /|\tau|^{2}$ as a harmonic function in $\left(y_{1}, y_{2}\right)$ such that the KK-6A brane solution takes exactly the same form as the D6-brane. Therefore the modular transformation $\tau \rightarrow-1 / \tau$ relating the two 6 -brane solutions is equivalent to a coordinate transformation in the base space. This transformation connects two points of the base with fibers S-dual to each other and therefore establishes the equivalence between the two 6-brane solutions. This is the reason why the D6-brane and the KK-6A brane do not have associated independent central charges in the Type IIB space-time supersymmetry algebra.

In the limit $A \rightarrow 0$, the MKK-monopole solution (6.3) yields the D7-brane solution with $\lambda=-\bar{\tau}$. Considering the compactification with a fiber modulus $-\tau^{-1}$ one obtains the NS-7B brane with $\lambda=\bar{\tau}^{-1}$. In particular the 7-brane solutions (3.31) and (3.33) correspond to the following choice of $\tau$ as a section of the base space $\left(x_{1}, x_{2}\right)$ :

$$
\tau_{\mathrm{D} 7}=\frac{1}{2 \pi i} \log \left(x_{2}-i x_{1}\right), \quad \tau_{\mathrm{NS} 7 \mathrm{~B}}=-\frac{1}{\tau_{\mathrm{D} 7}} .
$$

They can be written using the parameters of the torus-bundle (6.3). For the D7-brane we have

$$
\begin{aligned}
& d s_{\mathrm{D} 7}^{2}=\tau_{2}^{-1 / 2}\left(d s_{6,1}^{2}-d x_{3}^{2}\right)-R^{2} \tau_{2}^{-1 / 2}\left(d x_{1}^{2}+d x_{2}^{2}\right), \\
& e^{\phi}=\tau_{2}^{-1}, \quad C^{(0)}=-\tau_{1},
\end{aligned}
$$

and for the NS-7B brane

$$
\begin{aligned}
& d s_{\mathrm{NS} 7 \mathrm{~B}}^{2}=\left(\frac{\tau_{2}}{|\tau|^{2}}\right)^{-1 / 2}\left(d s_{6,1}^{2}-d x_{3}^{2}\right)-R^{2}\left(\frac{\tau_{2}}{|\tau|^{2}}\right)^{-1 / 2}\left(d x_{1}^{2}+d x_{2}^{2}\right), \\
& e^{\phi}=\left(\frac{\tau_{2}}{|\tau|^{2}}\right)^{-1}, \quad C^{(0)}=\frac{\tau_{1}}{|\tau|^{2}} .
\end{aligned}
$$

The base space becomes the transverse space of the 7-branes upon compactification and the $\mathrm{S}$-duality relation between the two branes can be traced back to the properties of the MKK-monopole interpreted as a torus-bundle. Namely, as we have seen above, the $\mathrm{S}$-duality mapping is just a gauge symmetry in the M-theory solution, in the sense that a coordinate transformation undoes the effect of the S-duality transformation. Thus, a coordinate transformation in the space transversal to the 7-branes relates both 7-brane solutions. As for the D6, KK6A brane solutions, this provides an explanation for the fact that the D7 and the NS-7B branes do not appear as independent branes in the Type IIB space-time supersymmetry algebra. 
The finite energy 7-brane solution of [21] is obtained from the monopole solution (6.3) by taking the modulus of the torus-bundle as

$$
j(\tau)=\frac{1}{x_{1}+i x_{2}},
$$

such that we identify all solutions related by a modular transformation in the sections. This defines $\tau$ as a holomorphic function, and yields the D7 (NS7B) brane solution in the weak (strong) coupling limit ${ }^{15}$.

If we now impose one extra isometry in the harmonic function $H, H=H\left(x_{1}\right)$, it is not possible to eliminate completely the dependence of the metric (6.3) on the coordinate $x_{2}$, since according to the relation between $H$ and $\boldsymbol{V}, V_{3}$ has to be a function of $x_{2}^{16}$. A particular type of this class of configurations has been interpreted in [41] as the compactification of M-theory giving rise to massive Type IIA string theory ${ }^{17}$ :

$$
d s_{\mathrm{MKK}}^{2}=d s_{6,1}^{2}-R^{2}\left(d x_{1}^{2}+d x_{2}^{2}\right)-\frac{A}{H}\left|d z+\left(m x_{2}+i H\right) d x_{3}\right|^{2} .
$$

This solution is obtained from (6.3) with the ansatz $\tau=m x_{2}+i H\left(x_{1}\right)$. We have substituted $\tau_{2}=H$ to make more clear the connection with the solution in [41]. The condition $\boldsymbol{\nabla} H=\boldsymbol{\nabla} \wedge \boldsymbol{V}$ fixes $H\left(x_{1}\right)=m\left|x_{1}\right|+$ const., so that (6.13) represents a domain-wall at $x_{1}=0$ with two different bundles at each side of the wall.

The limit $A \rightarrow 0$ yields the Scherk-Schwarz reduction of the Type IIB theory with $\lambda=-\bar{\tau}\left(x_{1}, x_{2}\right)$ as ansatz for the axion-dilaton modulus. Taking the limit $R \rightarrow 0$ as well, one obtains the T-dual theory, which is massive Type IIA string theory. Thus in order to obtain a D8-brane solution from (6.3), one requires a bundle $B$ with monodromy

$$
\left(\begin{array}{cc}
1 & m \\
0 & 1
\end{array}\right)
$$

in the zero volume limit $A \rightarrow 0, R \rightarrow 0$. From the point of view of F-theory, the Scherk-Schwarz reduction of Type IIB is a compactification on the 2-torus bundle over the circle in the $x_{2}$ direction (with $H$ depending on the other transverse direction $x_{1}$ ) for fixed area $A[41]$.

We know that T-duality along a transverse direction on the D7-brane gives the D8-brane [38]. This relation is also called massive T-duality and its precise form is given by the kind of Scherk-Schwarz reduction that is taken in the Type IIB side. This is determined by the monodromy (6.14) of the bundle $B$, so that it is possible to perform general Scherk-Schwarz reductions with a monodromy matrix spanning the whole $S L(2, \mathbb{Z})[13]$. These are related by $\mathrm{T}$-duality to different massive modifications of the Type IIA theory.

\footnotetext{
${ }^{15}$ After a $\pi / 2$ rotation in the base space.

${ }^{16}$ See Ref. [24] for a discussion about this point.

${ }^{17}$ In this reference this solution was interpreted as an M9-brane. However, we see here that it is just a Kaluza-Klein monopole with one extra isometry.
} 
If we perform a T-duality transformation in the direction $x_{2}$ transversal to the D7-brane, we obtain a D8-brane solution of the form

$$
\begin{aligned}
& d s_{\mathrm{D} 8}^{2}=\tau_{2}^{-1 / 2}\left(d s_{7,1}^{2}-R^{-2} \tau_{2} d x_{2}^{2}\right)-R^{2} \tau_{2}^{-1 / 2} d x_{1}^{2}, \\
& e^{\phi}=R^{-1} \tau_{2}^{-3 / 4}, \quad C^{(1)}=\left(\tau_{1}-m x_{2}\right) d x_{2} .
\end{aligned}
$$

For the T-duality the harmonic function $H$ must be taken as a function linear on $x_{1}$, hence $\tau_{1}=V_{3}$ must be linear in $x_{2}$. We choose $H=m\left|x_{1}\right|+$ const. and $V_{3}=m x_{2}$, and therefore $C^{(1)}=0$.

Similarly, one can consider the T-duality of the NS-7B brane. However, the metric associated to this solution depends, through the modulus of $\tau$, on the $x_{2}$ coordinate in which we are performing the T-duality transformation. Therefore we need to use the generalized Buscher T-duality rules derived in [13], which allow us to treat this case. The result is the KK-8A brane [13]:

$$
\begin{aligned}
& d s_{\mathrm{KK} 8 \mathrm{~A}}^{2}=\tau_{2}^{1 / 2}\left(d s_{7,1}^{2}-R^{-2} \tau_{2}^{-1} d x_{2}^{2}\right)-R^{2} \tau_{2}^{1 / 2} d x_{1}^{2}, \\
& e^{\phi}=R^{-1} \tau_{2}^{3 / 4}, \quad C^{(1)}=0 .
\end{aligned}
$$

Both solutions, the D8 and the KK-8A branes, can be obtained from the M9-brane solution of $[11]^{18}$ by dimensional reduction. We can interpret this M9-brane as a torus-bundle over the one-dimensional transverse space spanned by $x_{1}$,

$$
d s_{\mathrm{M} 9}^{2}=R^{2 / 3} d s_{7,1}^{2}-R^{-4 / 3} \tau_{2}^{-1}\left|d z+\tau\left(x_{1}\right) d x_{2}\right|^{2}-R^{8 / 3} d x_{1}^{2},
$$

with fiber modulus $\tau\left(x_{1}\right)=i H\left(x_{1}\right)$ and $R^{2}=H$. In this case the area of the torus is also a function of $x_{1}$. Reducing along $z$ we recover the D8-brane solution whereas the reduction along $x_{2}$ gives the KK-8A brane solution. The relation between this two reductions is an S-transformation of the section $\tau$. Analogously to the MKK-monopole solution, this modular transformation can be undone by a change of coordinates in the transverse space. First, let us rescale the metric so that $d s_{\mathrm{M} 9}^{2}$ takes the form

$$
d s_{\mathrm{M} 9}^{2}=d s_{7,1}^{2}-\frac{R^{-2}}{\tau_{2}}\left|d z+\tau d x_{2}\right|^{2}-R^{2} d x_{1}^{2} .
$$

In this form it is clear that the area of the torus $A=R^{-2}$, and therefore it is a function of $x_{1}$. The modular transformation $\tau_{2} \rightarrow \tau_{2}^{-1}$ can be generated by the change of coordinates

$$
d x_{1} \rightarrow \tau_{2} d x_{1}, \quad d z \rightarrow \tau_{2}^{-1} d z, \quad d x_{2} \rightarrow \tau_{2}^{-1} d x_{2} .
$$

We see that in this case it is also necessary to perform a change of coordinates in the torus metric, due to the dependence of its area on the transverse direction. Thus, a coordinate transformation relates the two 8-brane solutions, from where we can conclude that they represent the same physical object and therefore should have associated a single central charge in the Type IIA space-time supersymmetry algebra.

Notice that in (6.15) the most general solution compatible with $\boldsymbol{\nabla} H=\boldsymbol{\nabla} \wedge \boldsymbol{V}$ corresponds in fact to $V_{3}=m x_{2}+c$, with $c=$ constant, which gives a D8-brane with

\footnotetext{
${ }^{18}$ We take $\epsilon=-1$.
} 
$C^{(1)}=c d x_{2}$. This is indeed a solution to the massive IIA supergravity, and is related by dimensional reduction to a non-diagonal M9-brane solution. This non-diagonal M9-brane has the same form as in (6.17) but with fiber modulus $\tau=c+i H\left(x_{1}\right)$. Moreover, it can be obtained from $(6.17)$ by means of a T-transformation of the section, which induces a constant real part for $\tau$. This defines a 1-parameter family of (non-diagonal) M9-branes interpreted as a torus-bundle over the one-dimensional transverse space. Furthermore, it is easy to check that the non-diagonal M9-brane can be reduced to the usual diagonal one by redefining the $z$ coordinate. Thus it is also a solution of the massive 11-dimensional supergravity of [35].

The dimensional reduction of a non-diagonal M9-brane, with $\tau=c+i H$, gives rise to a D8-brane with $C^{(1)}=c d x_{2}$, and to a KK-8A brane:

$$
\begin{aligned}
& d s_{\mathrm{KK} 8 \mathrm{~A}}^{2}=\left(\frac{H}{c^{2}+H^{2}}\right)^{-1 / 2}\left(d s_{7,1}^{2}-R^{-2} \frac{H}{c^{2}+H^{2}} d x_{2}^{2}\right)-R^{2}\left(\frac{H}{c^{2}+H^{2}}\right)^{-1 / 2} d x_{1}^{2}, \\
& e^{\phi}=R^{-1}\left(\frac{H}{c^{2}+H^{2}}\right)^{-3 / 4}, \quad C^{(1)}=-\frac{c}{c^{2}+H^{2}} d x_{2} .
\end{aligned}
$$

This solution could be obtained as well by applying the massive T-duality rules of [13] to the NS-7B brane solution (6.11) with $\tau_{1}=m x_{2}+c$ and $\tau_{2}=H$. It is related to the D8-brane solution with $C^{(1)}=c d x_{2}$ by an S-transformation of the section $\tau=c+i H$.

\section{Conclusions}

We have constructed the world-volume effective action associated to the NS-7B brane and checked that it transforms as an $S L(2, \mathbb{Z})$ triplet together with the D7-brane. The third field entering in this triplet transformation is the electric-magnetic dual of the dilaton, which however does not have associated an independent 7-brane solution: the D7 and NS-7B brane solutions themselves are the ones carrying charge with respect to this field. We have clarified why the NS-7B brane does not appear in the Type IIB space-time supersymmetry algebra. From one point of view this brane is charged with respect to a field which is a combination of the duals of the axion and dilaton (with coefficients depending as well on the axion and dilaton), and therefore is not an independent field in the theory. This function is however non-local and this is why this field has to be introduced in the S-duality transformation rule.

From another point of view we have also shown that the D7 and NS7B brane solutions and the D6 and KK-6A brane solutions are related by a coordinate transformation in the two-dimensional transverse space which amounts to choosing a different region to parametrize the $S L(2, \mathbb{Z})$ moduli space. This provides further evidence for the fact that both 7-branes and 6-branes represent the same physical object. A similar kind of transformation relates the D8 and KK-8A brane solutions, in this case in the one-dimensional transverse space.

The relation

$$
d \tilde{C}^{(8)}=-C^{(0)} d B^{(8)}+|\lambda|^{2} d C^{(8)}
$$


between the field to which the NS-7B brane couples minimally and the duals of the axion and dilaton translates under T-duality into relationships between the fields to which the KK-6A and KK-8A branes couple minimally and the $C^{(7)}, C^{(9)}$ RR fields of the Type IIA theory. Namely,

$$
d\left(i_{k} N^{(8)}\right)=\left(i_{k} C^{(1)}\right) d\left(i_{k} B^{(8)}\right)-\left[\left(i_{k} C^{(1)}\right)^{2}+e^{-2 \phi} k^{2}\right] d C^{(7)}
$$

and

$$
d\left(i_{k} N^{(9)}\right)=\left(i_{k} C^{(1)}\right) d\left(i_{k} B^{(9)}\right)-\left[\left(i_{k} C^{(1)}\right)^{2}+e^{-2 \phi} k^{2}\right] d\left(i_{k} C^{(9)}\right) .
$$

The first expression gives a relation between the field $i_{k} N^{(8)}$, to which the KK-6A brane couples minimally, the RR 7-form and the T-dual of $B^{(8)}$, which we have assumed to be again an 8-form $B_{\mu_{1} \ldots \mu_{7} z}^{(8)^{\prime}}=B_{\mu_{1} \ldots \mu_{7} z}^{(8)}$. This 8-form in the Type IIA theory can be given some interpretation in connection to the M8-brane of [6,8]. This BPS 8-brane solution of M-theory is required by $\mathrm{U}$-duality of M-theory on $T^{8}$, but is however not predicted by the M-theory space-time supersymmetry algebra. An 8-brane in M-theory requires the existence of a 9-form potential to which it couples minimally. If we assume that the 11-dimensional background contains an isometry (say in the direction $z$ ), along which we reduce to go to the Type IIA theory, this 9 -form potential could be the electric-magnetic dual of the component of the metric $\hat{g}_{z z}$. Then the 9-form would be interpreted as the dual of the dilaton at strong coupling. When reducing to ten dimensions it would give rise to a 9-form and an 8-form, which would have associated an 8-brane and a 7-brane respectively. The 8 -form could be the $B^{(8)}$ field above and the 9-form the $B^{(9)}$ field that appears in (7.3), and which comes as well from the T-duality of the field $B^{(8)}$ of the Type IIB theory: $B_{\mu_{1} \ldots \mu_{8}}^{(8)^{\prime}}=B_{\mu_{1} \ldots \mu_{8} z}^{(9)}$. These two fields can be interpreted in the Type IIA theory as the dual of the dilaton, for $B^{(8)}$, and the dual of a mass parameter, for $B^{(9)}$. A 7-brane and an 8-brane associated to these fields are however not predicted by the Type IIA space-time supersymmetry algebra. It is unclear to us at this point why this happens. One possibility is that it is the D6, KK6A and D8, KK8A branes themselves the ones carrying charge with respect to the fields $i_{k} B^{(8)}$ and $B^{(9)}$ respectively, as happens with the 7-branes and the $B^{(8)}$ field in the Type IIB theory. The two relations (7.2) and (7.3) suggest that the fields to which the KK-6A and the KK-8A branes couple are not independent fields in the theory, and this would explain why these branes do not appear in the Type IIA space-time supersymmetry algebra. One way to clarify further this issue would be to analyze the equations of motion associated to the fields involved in these relations, i.e. the RR 1 -form, the dilaton and the $k^{2}$ field, to elucidate which are the independent dual potentials in the theory. We hope to report progress in this direction in the near future. Finally, T-duality in (7.2) also establishes a relationship between the field $\left(i_{k} i_{h} N^{(9)}\right)$ to which the KK-7B brane couples minimally and $i_{h} C^{(8)}$ (in the truncation $B^{(2)}=C^{(2)}=0$ ). As before, this could be the reason for the non-occurrence of the KK-7B brane in the Type IIB space-time supersymmetry algebra.

There are other exotic branes required to fill up multiplets of BPS states of M-theory on $T^{d}$ for $d \geqslant 8$ which we have not considered in this paper [6-10]. Some of them are very simply related to the branes that we have studied, and we have indeed checked that the duality relations that we mention below hold with the world-volume effective actions that we have constructed. For instance T-duality of the KK-6A brane along a world- 
volume direction gives a 5-brane in Type IIB with two Killing isometries, which in the notation of [8] corresponds to the $\left(5,2^{2} ; 3\right)$-brane. This brane forms an S-duality doublet with the $\left(5,2^{2} ; 2\right)$-brane obtained by T-duality of the Type IIA KK-monopole along a transverse direction. Similarly, T-duality of the KK-8A brane along the transverse direction gives a 7-brane in Type IIB with two Killing directions, which in the notation of $[8]$ corresponds to a $\left(7,2^{3} ; 3\right)$ brane. This brane forms an S-duality doublet with the $\left(7,2^{3} ; 4\right)$ brane obtained from T-duality of the NS-9A brane along a world-volume direction [8]. Therefore in this case the two Killing directions are interpreted as world-volume directions, and the brane as a 9-brane.

NS-9A branes seem to play a role in the description of the heterotic $E_{8} \times E_{8}$ as a non-perturbative orientifold of the Type IIA theory [3]. In this description the gauge group arises in the form of Chan-Paton factors of (dimensionally reduced) open M2-branes ending on the $8(+8)$ M9-branes which are positioned on top of the two orientifold fixed planes, associated to the $I_{10} \Omega_{M}$ symmetry of M-theory (here $I_{10}$ is the inversion of the eleventh direction and $\Omega_{M}$ reverses the sign of the three form of 11-dimensional supergravity). In this description $\Omega_{M}$ is interpreted as the world-volume operation reversing one of the spatial directions of the M2-brane. A T-duality transformation (along a world-volume direction) in this construction seems to indicate that the heterotic $\mathrm{SO}(32)$ could be obtained as an orientifold of F-theory on $T^{2}$ divided by the symmetry $I_{10,11} \Omega_{F}$, with $\Omega_{F}=T \Omega_{M} T^{-1}$ ( $T$ denotes the T-duality transformation). T-duality predicts that the $\left(7,2^{3} ; 4\right)$-branes above would be the ones responsible for the gauge structure. It would be interesting to explore this connection in more detail and see in particular how the $\Omega_{F}$ symmetry could be defined on the F-theory side.

\section{Acknowledgements}

We would like to thank A. Arrizabalaga, E. Bergshoeff, M. de Roo, C. Hull, N. Obers and T. Ortín for useful discussions. E.E would like to thank the String Theory group of Napoli University and especially F. Pezzella, for hospitality. He also thanks the people of the Spinoza Institute for hospitality.

\section{Appendix A}

In this section we give the gauge transformation rules of the new fields that couple to the effective actions of the branes considered in the paper. The notation is that of [17], where the transformation rules of the fields coupled to D $p$-branes, NS5-branes and Kaluza-Klein monopoles can also be found.

The field to which the KK-6A brane couples minimally transforms as

$$
\begin{aligned}
\delta\left(i_{k} N^{(8)}\right)= & 7\left\{\partial\left(i_{k} \Omega^{(7)}\right)-15 \partial\left(i_{k} \tilde{\Lambda}\right)\left(i_{k} C^{(3)}\right)+30 \partial \Lambda^{(2)}\left(i_{k} C^{(3)}\right)^{2}\right. \\
& \left.-20 C^{(3)}\left(i_{k} C^{(3)}\right) \partial\left(i_{k} \Lambda^{(2)}\right)-\left(i_{k} N^{(7)}\right) \partial \Lambda^{(0)}\right\} .
\end{aligned}
$$


The field $i_{k} N^{(9)}$ coupled to the KK-8A brane transforms as

$$
\begin{aligned}
\delta\left(i_{k} N^{(9)}\right)= & \left\{\partial\left(i_{k} \Omega^{(8)}\right)+21 \partial\left(i_{k} \Sigma^{(6)}\right)\left[\left(i_{k} C^{(3)}\right)+2\left(i_{k} B^{(2)}\right)\left(D X C^{(1)}\right)\right]\right. \\
& +105 \partial\left(i_{k} \Lambda^{(4)}\right)\left(i_{k} C^{(3)}\right)\left[\left(i_{k} C^{(3)}\right)+4\left(i_{k} B^{(2)}\right)\left(D X C^{(1)}\right)\right] \\
& +315\left(i_{k} C^{(3)}\right)^{2} \partial\left(i_{k} \Lambda^{(2)}\right) D X D X B^{(2)} \\
& +\frac{7 !}{4}\left(i_{k} C^{(3)}\right) \partial\left(i_{k} \Lambda^{(2)}\right) B^{(2)}\left(i_{k} B^{(2)}\right)\left(D X C^{(1)}\right) \\
& -\left(2 \pi \alpha^{\prime}\right)\left[i_{k} N^{(8)}+7\left(i_{k} N^{(7)}\right)\left(D X C^{(1)}\right)\right. \\
& +35 D X D X D X C^{(3)}\left(i_{k} C^{(3)}\right)^{2}+70\left(2 C^{(3)}\left(i_{k} B^{(2)}\right)\right. \\
& \left.\left.\left.+3\left(i_{k} C^{(3)}\right) B^{(2)}\right)\left(i_{k} C^{(3)}\right)\left(D X C^{(1)}\right)\right] \partial\left(i_{k} \Lambda\right)\right\} .
\end{aligned}
$$

In the KK-7B brane $\left(i_{h} i_{k} N^{(9)}\right)$ transforms as

$$
\begin{aligned}
\delta\left(i_{h} i_{k} N^{(9)}\right)= & 7\left\{\partial\left(i_{h} i_{k} \Omega^{(8)}\right)+6\left(i_{h} i_{k} \mathscr{N}^{(7)}\right) \partial\left(i_{h} i_{k} \Lambda^{(3)}\right)\right. \\
& \left.-15 \partial\left(i_{h} \Lambda^{(3)}\right)\left(i_{h} i_{k} C^{(4)}\right)^{2}+10 \partial\left(i_{h} i_{k} \Lambda^{(3)}\right)\left(i_{h} C^{(4)}\right)\left(i_{h} i_{k} C^{(4)}\right)\right\} .
\end{aligned}
$$

This is the correct gauge transformation rule in the truncation $B^{(2)}=C^{(2)}=0$. Also, in this truncation:

$$
\begin{aligned}
\delta\left(i_{h} i_{k} N^{(8)}\right) & =6 \partial\left(i_{h} i_{k} \Omega^{(7)}\right), \\
\delta\left(i_{h} i_{k} \mathscr{N}^{(8)}\right) & =6 \partial\left(i_{h} i_{k} \tilde{\Omega}^{(7)}\right) . \\
N^{(9)}, N^{(8)}, \mathscr{N}^{(8)} & \text { are now 9- and 8-forms in the Type IIB theory. }
\end{aligned}
$$

\section{References}

[1] C.M. Hull, Nucl. Phys. B 509 (1998) 216. hep-th/9705162.

[2] P.K. Townsend, M-theory from its Superalgebra, Cargèse Lectures 1997, hep-th/9712004.

[3] E. Bergshoeff, E. Eyras, R. Halbersma, C.M. Hull, Y. Lozano, J.P. van der Schaar, Nucl. Phys. B 564 (2000) 29. hep-th/9812224.

[4] E. Bergshoeff, M. de Roo, B. Janssen, T. Ortín, Nucl. Phys. B 550 (1999) 289. hep-th/9901055.

[5] C.M. Hull, Phys. Lett. B 462 (1999) 271. hep-th/9812210.

[6] S. Elitzur, A. Giveon, D. Kutasov, E. Rabinovici, Nucl. Phys. B 509 (1998) 122. hep-th/9707217.

[7] M. Blau, M. O’Loughlin, Nucl. Phys. B 525 (1998) 182. hep-th/9712047.

[8] C.M. Hull, J. High Energy Phys. 07 (1998) 018. hep-th/9712075.

[9] N.A. Obers, B. Pioline, E. Rabinovici, Nucl. Phys. B 525 (1998) 163. hep-th/9712084.

[10] N.A. Obers, B. Pioline, Phys. Rep. 318 (1999) 113. hep-th/9809039.

[11] E. Bergshoeff, J.P. van der Schaar, Class. Quant. Grav. 16 (1999) 23. hep-th/9806069.

[12] E. Eyras, Y. Lozano, Brane actions and string dualities, hep-th/9812225.

[13] P. Meessen, T. Ortín, Nucl. Phys. B 541 (1999) 195. hep-th/9806120.

[14] A.A. Tseytlin, Nucl. Phys. B 469 (1996) 51. hep-th/9602064. 
[15] Y. Lozano, Phys. Lett. B 399 (1997) 233. hep-th/9701186.

[16] M. Aganagic, J. Park, C. Popescu, J.H. Schwarz, Nucl. Phys. B 496 (1997) 215. hep-th/9702133.

[17] E. Eyras, B. Janssen, Y. Lozano, Nucl. Phys. B 531 (1998) 275. hep-th/9806169.

[18] M.R. Douglas, M. Li, D-brane realization of $\mathscr{N}=2$ super Yang-Mills theory in four dimensions, hep-th/9604041.

[19] G. Dall'Agata, K. Lechner, M. Tonin, J. High Energy Phys. 07 (1998) 017. hep-th/9806140.

[20] M. Cederwall, A. von Gussich, B.E.W. Nilsson, P. Sundell, A. Westerberg, Nucl. Phys. B 490 (1997) 179. hep-th/9611159.

[21] G.W. Gibbons, M.B. Green, M.J. Perry, Phys. Lett. B 370 (1996) 37. hep-th/9511080.

[22] E. Bergshoeff, B. Janssen, T. Ortín, Phys. Lett. B 410 (1997) 132. hep-th/9706117.

[23] E. Bergshoeff, E. Eyras, Y. Lozano, Phys. Lett. B 430 (1998) 77. hep-th/9802199.

[24] E. Bergshoeff, M. de Roo, E. Eyras, B. Janssen, J.P. van der Schaar, Class. Quant. Grav. 14 (1997) 2757. hep-th/9704120.

[25] P.K. Townsend, Phys. Lett. B 409 (1997) 131. hep-th/9705160.

[26] C. Vafa, Nucl. Phys. B 469 (1996) 403. hep-th/9602022.

[27] A. Dabholkar, Lectures on orientifolds and duality, hep-th/9804208.

[28] B.R. Greene, A. Shapere, C. Vafa, S.T. Yau, Nucl. Phys. B 337 (1990) 1.

[29] A. Sen, Nucl. Phys. B 475 (1996) 562. hep-th/9605150.

[30] K. Dasgupta, S. Mukhi, Phys. Lett. B 385 (1996) 125. hep-th/9606044.

[31] A. Johansen, Phys. Lett. B 395 (1997) 36. hep-th/9608186.

[32] M.R. Gaberdiel, B. Zwiebach, Nucl. Phys. B 518 (1998) 151. hep-th/9709013.

[33] M.R. Gaberdiel, T. Hauer, B. Zwiebach, Nucl. Phys. B 525 (1998) 117. hep-th/9801205.

[34] D. O'Driscoll, Phys. Lett. B 454 (1999) 240. hep-th/9901028.

[35] E. Bergshoeff, Y. Lozano, T. Ortín, Nucl. Phys. B 518 (1998) 363. hep-th/9712115.

[36] K. Bautier, S. Deser, M. Henneaux, D. Seminara, Phys. Lett. B 406 (1997) 49. hep-th/9704131.

[37] L.J. Romans, Phys. Lett. B 169 (1986) 374.

[38] E. Bergshoeff, M. de Roo, M.B. Green, G. Papadopoulos, P.K. Townsend, Nucl. Phys. B 470 (1996) 113. hep-th/9601150.

[39] M. de Roo, Intersecting Branes and Supersymmetry, Presented at Supersymmetry and Quantum Field Theory, Int. Seminar dedicated to the memory of D.V. Volkov, Kharkov, 1997, hep-th/9703124.

[40] E. Bergshoeff, J. Gomis, P.K. Townsend, Phys. Lett. B 421 (1998) 109. hep-th/9711043.

[41] C.M. Hull, J. High Energy Phys. 9811 (1998) 027. hep-th/9811021. 